COMMUNICATIONS IN

ANALYSIS AND GEOMETRY

Volume 6, Number 4, 633-668, 1998

\title{
Weierstrass type representation of harmonic maps into symmetric spaces ${ }^{1}$
}

\author{
J. Dorfmeister ${ }^{2}$, F. Pedit AND H. Wu
}

\section{Introduction.}

Over the past five years substantial progress has been made in the understanding of harmonic maps $f: M \rightarrow G / K$ of a compact Riemann surface $M$ into a compact symmetric space $G / K[29,30,12,14,17,22,5,7,10,31]$. When $M$ is the Riemann sphere all such maps may be obtained from holomorphic curves into some associated twistor space [11, 29, 14]. In contrast, if $M$ is a 2-torus, then every harmonic map $\phi: M \rightarrow G / K$ (satisfying specific nondegeneracy assumptions) may be obtained from a solution to a certain family of completely integrable finite dimensional systems of Hamiltonian ODE in Lax form on a loop algebra [5, 7, 17]. Such harmonic maps are called harmonic maps of finite type and, e.g., all harmonic 2-tori in $S^{n}, \mathbb{C} P^{n}$ are accounted for in this way [4]. So far there is no comparable systematic theory when $M$ has higher genus. Nevertheless there are examples: the harmonic maps $f: M \rightarrow S^{2}$ arising as Gauss maps of Kapouleas' [18, 19] constant mean curvature surfaces of genus $g \geq 2$ and the minimal (and hence harmonic) maps $f: M \rightarrow S^{3}$ given by Lawson's [20] minimal surfaces of genus $g \geq 2$.

The objective of the present paper is to develop a systematic scheme for the construction of all harmonic maps $f: M \rightarrow G / K$ when $M$ is simply connected. Since the case where $M$ is the Riemann sphere has been accounted for completely in the work of Uhlenbeck [29], we think of $M$ as the universal cover of some compact Riemann surface of genus $\geq 1$. Our method has its origins in what is known as the Symes formula for integrating Lax pair equations [28]. Analogs of this formula have been used to construct (extended solutions of) harmonic 2-spheres in Lie groups [14] and to construct

\footnotetext{
${ }^{1}$ Partially supported by NSF grant DMS-9205293 and SFB 288 at Technische Universität Berlin.

${ }^{2}$ Partially supported by KITCS grant OSR-9255223.
} 
(extended lifts of) finite type harmonic maps in compact symmetric spaces $G / K[10,7]$. The harmonic maps so obtained have the property that the $(1,0)$-part of the derivative, $\partial f$, takes values in a single adjoint orbit.

A standard holomorphic differential argument, namely Ad-invariant polynomials on the Lie algebra $\mathfrak{g}$ of $G$ evaluated on $\partial f$ provide holomorphic differentials on $M$, together with a nondegeneracy assumption, ensure this orbit condition when $M$ is the 2 -sphere or a 2-torus. This is the starting point for the analysis of harmonic 2-tori in symmetric spaces in [5].

Since on a genus $\geq 2$ Riemann surface $M$ these holomorphic differentials may have isolated zeros, the derivative $\partial f$ no longer stays in a fixed level set of Ad-invariant polynomials and thus such a harmonic map $f: M \rightarrow G / K$ cannot, in general, be of finite type. In the simplest case where $G / K=\boldsymbol{S U}(2) / S^{1}$ is the 2-sphere the Killing form (, ) is (up to scale) the only invariant polynomial. Thus, for a harmonic map $f: M \rightarrow S^{2}$, either $(\partial f, \partial f) \equiv 0$, in which case $f$ is \pm holomorphic (this is always the case if $M$ has genus 0$)$ and $\partial f$ is nilpotent, or $(\partial f, \partial f)$ has isolated zeros and away from these zeros $\partial f$ is regular semisimple. If $M$ has genus one $(\partial f, \partial f)$ is nowhere zero, i.e., $f$ is nowhere conformal, $\partial f$ is regular semisimple and thus of finite type by the results in [5]. Over a genus $\geq 2$ Riemann surface $\partial f$ will change orbit type at isolated points.

To account for such a behavior we use a Weierstrass-type representation for harmonic maps $f: M \rightarrow G / K$ which results from a variation of the Symes formula. We start with a holomorphic 1-form $\mu=\sum_{i \geq-1} \lambda^{i} \mu_{i}$ taking values in a subspace of a twisted loop algebra. Integrating $g^{-1} d g=\mu$ over the (simply connected) Riemann surface $M$ gives a map $g$ with values in a corresponding twisted loop group. Such loop groups allow decompositions of Iwasawa type into real loops and loops extending holomorphically to the interior of the $\lambda$-disc. Factorization of $g$ according to this decomposition,

$$
g=F b,
$$

yields an (extended) frame of the harmonic map $f=\pi \circ F_{\left.\right|_{\lambda=1}}: M \rightarrow G / K$ whose derivative is given by

$$
\partial f=\operatorname{Ad} F_{\left.\right|_{\lambda=1}}\left(\mu_{-1}\right) .
$$

Conversely we prove that, if $M$ is not the Riemann sphere $S^{2}$, every harmonic map $f: M \rightarrow G / K$ is obtained from some holomorphic 1-form $\mu=\sum_{i \geq-1} \lambda^{i} \mu_{i}$ on the universal covering. If $\mu=\eta d z$ for some suitable constant $\eta=\sum_{k>-1} \lambda^{k} \eta_{k}$ we recover the finite type harmonic maps of [5]. Finally, using a Riemann-Hilbert splitting we show that any harmonic map 
$f: M \rightarrow G / K$ may be obtained by the above construction from a meromorphic 1-form $\mu=\lambda^{-1} \eta$ where $\eta$ takes values in the finite dimensional Lie algebra $\mathfrak{g}^{\mathbb{C}}$. The set of poles of $\eta$ is given as the locus where the RiemannHilbert factorization fails to exist. In a certain way this justifies to view the meromorphic 1-forms $\mu$ as Weierstrass-type data for harmonic maps.

We now give a brief description of the contents of the various sections in this paper: we start in section 2 by introducing the necessary loop groups of maps $g: S^{1} \rightarrow G$ where $G$ is a compact semisimple Lie group. Using existing factorization theorems [23] we adopt these results to the twisted setting, i.e., spaces of loops $g: S^{1} \rightarrow G$ which have $g\left(e^{2 \pi i / k} \lambda\right)=\sigma g(\lambda)$ for some order $k$ automorphism $\sigma$ of $G$. Further, we review the Grassmannian model, the determinant line bundle and its relation to the loop group factorizations. This section, containing mostly extensions to the twisted setting of material well documented, has more the flavor of an appendix. Since it also contains some basic setups and notations used throughout the paper we decided to leave it as an initial section. Crucial for the understanding of the paper is mainly the material up to theorem 2.3 and lemma 2.6. We suggest that the Reader, after familiarizing himself with the basic notational conventions, skips this section altogether and only goes back to it when prompted in the paper. The technicalities in this section are well-known to the expert and non-experts on this subject do not need to go into them to understand the geometrical constructions carried out in the paper.

In section 3 we reformulate the harmonic map equations for maps $f: M \rightarrow G / K$ of a Riemann surface $M$ into a compact symmetric space $G / K$ as the flatness of a certain loop algebra valued 1-form. The zero curvature equations in this case are the Yang-Mills-Higgs equations [16, 7]. Integrating this loop of 1-forms gives a loop of frames $F_{\lambda}: M \rightarrow G$ of harmonic maps $f_{\lambda}: M \rightarrow G / K$, i.e., $f_{\lambda}=\pi \circ F_{\lambda}, \pi: G \rightarrow G / K$ the coset projection, with $f=f_{1}$, which we call, following Uhlenbeck [29], an extended frame (or lift).

Section 4 deals with the construction of extended frames using the loop group factorizations discussed in section 2 together with a version of the Symes method as described above. We relate our approach to the construction of finite type harmonic maps [5], the Adler-Kostant-Symes scheme [1, 7] and the $r$-matrix scheme. Our construction, assigning an extended frame to a holomorphic 1 -form $\mu=\sum_{i \geq-1} \lambda^{i} \mu_{i}$ is equivariant with respect to natural loop group actions by dressing on the extended frames and by gauge transformations on the 1-forms $\mu$. We briefly discuss how this can be used to study dressing orbits of harmonic maps [6] in the spirit of Segal-Wilson's study of the KdV equation [26]. We conclude the paper by applying the 
general theory to the special case of harmonic maps $f: M \rightarrow S^{2}$, the Gauss maps of constant mean curvature surfaces in $\mathbb{R}^{3}$.

Parts of this work were done while various of the authors visited the SFB 288 at Technische Universität Berlin and the KITCS at the University of Kansas. We thank the members of both institutions for their interest and support in this project. We also wish to thank Fran Burstall for his many discussions and suggestions of improvements while the paper was written. The pictures at the end of the paper were produced using the "Mr. Bubble" module written by Charlie Gunn at SFB-288 TU-Berlin.

\section{Loop groups, Grassmannians and the $\tau$-function.}

The main technical tool for our study of harmonic maps will be the existence of various loop group factorizations. We will give a self-contained overview of the basic results, referring the reader to [23] for details and derive the factorization theorems we need from there. So let us fix the following ingredients:

(i) a compact (connected) semisimple Lie group $G$ with Lie algebra $\mathfrak{g}$,

(ii) an automorphism $\sigma: G \rightarrow G$ of order $k$ and fixed point set $G^{\sigma}=K$. We denote the induced automorphism on $\mathfrak{g}$ again by $\sigma$, so that $\mathfrak{g}^{\sigma}=\mathfrak{k}$ is the Lie algebra of $K$,

(iii) the primitive $k$-th root of unity $\omega=e^{2 \pi i / k}$,

(iv) an Iwasawa decomposition $K^{\mathbb{C}}=K \cdot B, K \cap B=\{e\}$ where $B \subset K^{\mathbb{C}}$ is a solvable subgroup, with corresponding decomposition $\mathfrak{k}^{\mathbb{C}}=\mathfrak{k} \oplus \mathfrak{b}$.

With this at hand, we define

$$
\Lambda G_{\sigma}^{\mathbb{C}}=\left\{g: S^{1} \rightarrow G^{\mathbb{C}} ; \quad g(\omega \lambda)=\sigma g(\lambda), \quad \lambda \in S^{1}\right\}
$$

and equip it with some $H^{s}$-topology for $s>1 / 2$. Then $\Lambda G_{\sigma}^{\mathbb{C}}$ becomes a complex Banach Lie group with Lie algebra

$$
\Lambda \mathfrak{g}_{\sigma}^{\mathbb{C}}=\left\{\xi: S^{1} \rightarrow \mathfrak{g}^{\mathbb{C}} ; \quad \xi(\omega \lambda)=\sigma \xi(\lambda), \quad \xi \text { is } H^{s}-\operatorname{smooth}\right\} .
$$

$\Lambda G_{\sigma}^{\mathbb{C}}$ has several closed Lie subgroups of importance to us: the real form

$$
\Lambda G_{\sigma}=\left\{g \in \Lambda G_{\sigma}^{\mathbb{C}} ; \quad g(\lambda) \in G, \quad \lambda \in S^{1}\right\}
$$


the loops extending holomorphically to the interior of the unit disc $D=$ $\{\lambda \in \mathbb{C} ;|\lambda|<1\}$ taking values in a fixed subgroup $H \subset K^{\mathbb{C}}$ at $\lambda=0$,

$$
\Lambda_{H}^{+} G_{\sigma}^{\mathbb{C}}=\left\{g \in \Lambda G_{\sigma}^{\mathbb{C}} ; g \text { extends holomorphically to } D, g(0) \in H\right\}
$$

and the loops extending holomorphically to the exterior of the unit disc $E=\{\lambda \in \mathbb{C} ; \lambda>1\}$ taking values in a fixed subgroup $H \subset K^{\mathbb{C}}$ at $\lambda=\infty$,

$$
\Lambda_{H}^{-} G_{\sigma}^{\mathbb{C}}=\left\{g \in \Lambda G_{\sigma}^{\mathbb{C}} ; g \text { extends holomorphically the } E, g(\infty) \in H\right\} .
$$

In case $H=K^{\mathbb{C}}$ we omit the subscript $H$ and we use the subscript $*$ for $H=\{e\}$, in which case we deal with based loops.

Denote the $\omega^{i}$-eigenspace of $\sigma$ on $\mathfrak{g}^{\mathbb{C}}$ by $\mathfrak{g}_{i}$. Clearly, $\mathfrak{g}_{0}=\mathfrak{k}^{\mathbb{C}}, \mathfrak{g}_{-i}=\overline{\mathfrak{g}_{i}}$ and $\left[\mathfrak{g}_{i}, \mathfrak{g}_{j}\right] \subseteq \mathfrak{g}_{i+j}$ (with arithmetic modulo $k$ ), where conjugation is always with respect to the real form $\mathfrak{g}$. Then, if $\xi \in \Lambda \mathfrak{g}_{\sigma}^{\mathbb{C}}$, its Fourier decomposition is

$$
\xi=\sum_{i \in \mathbb{Z}} \lambda^{i} \xi_{i}, \quad \xi_{i} \in \mathfrak{g}_{i}
$$

and the (closed) Lie subalgebras of $\Lambda \mathfrak{g}_{\sigma}^{\mathbb{C}}$ corresponding to the subgroups $\Lambda G_{\sigma}, \Lambda_{H}^{+} G_{\sigma}^{\mathbb{C}}, \Lambda_{H}^{-} G_{\sigma}^{\mathbb{C}}$ are

$$
\begin{aligned}
\Lambda \mathfrak{g}_{\sigma} & =\left\{\xi \in \Lambda \mathfrak{g}_{\sigma}^{\mathbb{C}} ; \quad \xi_{-i}=\bar{\xi}_{i}\right\}, \\
\Lambda_{\mathfrak{h}}^{+} \mathfrak{g}_{\sigma}^{\mathbb{C}} & =\left\{\xi \in \Lambda \mathfrak{g}_{\sigma}^{\mathbb{C}} ; \quad \xi_{i}=0 \text { for } i<0, \quad \xi_{0} \in \mathfrak{h}\right\}, \\
\Lambda_{\mathfrak{h}}^{-} \mathfrak{g}_{\sigma}^{\mathbb{C}} & =\left\{\xi \in \Lambda \mathfrak{g}_{\sigma}^{\mathbb{C}} ; \quad \xi_{i}=0 \text { for } i>0, \quad \xi_{0} \in \mathfrak{h}\right\},
\end{aligned}
$$

where $\mathfrak{h}$ is the Lie algebra of $H$. If $\xi \in \Lambda \mathfrak{g}_{\sigma}^{\mathbb{C}}$ then $\xi=\xi^{-}+\xi^{0}+\xi^{+}$where

$$
\xi^{-}=\sum_{i<0} \lambda^{i} \xi_{i}, \quad \xi^{0}=\xi_{0} \in \mathfrak{k}^{\mathbb{C}} \text { and } \xi^{+}=\sum_{i>0} \lambda^{i} \xi_{i} .
$$

For $\eta \in \mathfrak{k}^{\mathbb{C}}$ let $\eta_{\mathfrak{k}}$ and $\eta_{\mathfrak{b}}$ denote the projections along $\mathfrak{k}^{\mathbb{C}}=\mathfrak{k} \oplus \mathfrak{b}$. Then we have

$$
\begin{aligned}
\xi & =\left(\xi^{-}+\overline{\xi^{-}}+\left(\xi^{0}\right)_{\mathfrak{k}}\right)+\left(\xi^{+}-\overline{\xi^{-}}+\left(\xi^{0}\right)_{\mathfrak{b}}\right) \\
& =\xi^{-}+\left(\xi^{0}+\xi^{+}\right) \\
& =\left(\xi^{-}+\xi^{0}\right)+\xi^{+}
\end{aligned}
$$

where conjugation is with respect to the real form $\Lambda \mathfrak{g}_{\sigma}$, i.e., $\bar{\xi}=\sum \lambda^{i} \bar{\xi}_{-i}$. From this we immediately obtain 


\section{Lemma 2.1.}

$$
\begin{gathered}
\Lambda \mathfrak{g}_{\sigma}^{\mathbb{C}}=\Lambda \mathfrak{g}_{\sigma} \oplus \Lambda_{\mathfrak{b}}^{+} \mathfrak{g}_{\sigma}^{\mathbb{C}} \\
\Lambda \mathfrak{g}_{\sigma}^{\mathbb{C}}=\Lambda_{*}^{-} \mathfrak{g}_{\sigma}^{\mathbb{C}} \oplus \Lambda^{+} \mathfrak{g}_{\sigma}^{\mathbb{C}}=\Lambda^{-} \mathfrak{g}_{\sigma}^{\mathbb{C}} \oplus \Lambda_{*}^{+} \mathfrak{g}_{\sigma}^{\mathbb{C}}
\end{gathered}
$$

In what follows we will need global analogs of the above decompositions.

Let us denote by $\Lambda G^{\mathbb{C}}, \Lambda_{H}^{+} G^{\mathbb{C}}, \Lambda_{H}^{-} G^{\mathbb{C}}$ and $\Lambda G$ the groups $\Lambda G_{\sigma}^{\mathbb{C}}$ etc. for $\sigma=$ id and let $G^{\mathbb{C}}=G \cdot \widetilde{B}$ be an Iwasawa decomposition of $G^{\mathbb{C}}$. Then Pressley-Segal [23] prove the following factorization results (Pressley and Segal consider smooth loops but remark that the result holds for $H^{s}$-loops, $s>\frac{1}{2}$, as well):

Theorem 2.2. $\quad$ (i) Multiplication $\Lambda G \times \Lambda_{\mathscr{B}}^{+} G^{\mathbb{C}} \rightarrow \Lambda G^{\mathbb{C}}$ is a diffeomorphism onto. In particular, every $g \in \Lambda G^{\mathbb{C}}$ has a unique decomposition

$$
g=a b
$$

where $a \in \Lambda G, b \in \Lambda_{\mathbb{B}}^{+} G^{\mathbb{C}}$.

(ii) Multiplication $\Lambda_{*}^{-} G^{\mathbb{C}} \times \Lambda^{+} G^{\mathbb{C}} \rightarrow \Lambda G^{\mathbb{C}}$ is a diffeomorphism onto the open and dense subset $\Lambda_{*}^{-} G^{\mathbb{C}} \cdot \Lambda^{+} G^{\mathbb{C}}$, called the "big cell". In particular, if $g \in \Lambda G^{\mathbb{C}}$ is contained in the big cell then $g$ has a unique decomposition

$$
g=g_{-} g_{+},
$$

where $g_{-} \in \Lambda_{*}^{-} G^{\mathbb{C}}$ and $g_{+} \in \Lambda^{+} G^{\mathbb{C}}$. The analogous result holds for the multiplication map $\Lambda^{-} G^{\mathbb{C}} \times \Lambda_{*}^{+} G^{\mathbb{C}} \rightarrow \Lambda G^{\mathbb{C}}$.

From this we can relatively easily deduce the twisted loop group factorizations corresponding to the Lie algebra decompositions in Lemma 2.1.

Theorem 2.3. $\quad$ (i) Multiplication $\Lambda G_{\sigma} \times \Lambda_{B}^{+} G_{\sigma}^{\mathbb{C}} \rightarrow \Lambda G_{\sigma}^{\mathbb{C}}$ is a diffeomorphism onto.

(ii) Multiplication $\Lambda_{*}^{-} G_{\sigma}^{\mathbb{C}} \times \Lambda^{+} G_{\sigma}^{\mathbb{C}} \rightarrow \Lambda G_{\sigma}^{\mathbb{C}}$ is a diffeomorphism onto the open and dense set $\Lambda_{*}^{-} G_{\sigma}^{\mathbb{C}} \cdot \Lambda^{+} G_{\sigma}^{\mathbb{C}}$, again called the big cell. The analogous result holds for the multiplication map $\Lambda^{-} G_{\sigma}^{\mathbb{C}} \times \Lambda_{*}^{+} G_{\sigma}^{\mathbb{C}} \rightarrow \Lambda G_{\sigma}^{\mathbb{C}}$.

Proof. (i) Since the multiplication map of Theorem 2.2 (i) restricts to a diffeomorphism $\Lambda G_{\sigma} \times \Lambda^{+} G_{\sigma}^{\mathbb{C}} \rightarrow \Lambda G_{\sigma}^{\mathbb{C}}$ into, it remains to verify the surjectivity 
of this map. If $g \in \Lambda G_{\sigma}^{\mathbb{C}}$ then by Theorem 2.2 (i) $g=a b$ with $a \in \Lambda G$ and $b \in \Lambda_{\widetilde{B}}^{+} G^{\mathbb{C}}$. Thus, letting $(\sigma g)(\lambda)=\sigma\left(g\left(\omega^{-1} \lambda\right)\right)$,

$$
a b=g=\sigma(g)=\sigma(a) \sigma(b)
$$

and, since $\sigma(\Lambda G) \subseteq \Lambda G, \sigma\left(\Lambda^{+} G^{\mathbb{C}}\right) \subseteq \Lambda^{+} G^{\mathbb{C}}$ and $\Lambda G \cap \Lambda^{+} G^{\mathbb{C}}=G$, we obtain

$$
a^{-1} \sigma(a)=b \sigma(b)^{-1}=u \in G
$$

so that

$$
\sigma(a)=a u, \quad \sigma(b)=u^{-1} b .
$$

Using the unique decomposition $\Lambda^{+} G^{\mathbb{C}}=G^{\mathbb{C}} \cdot \Lambda_{*}^{+} G^{\mathbb{C}}$ we write

$$
b=b_{0} h
$$

and get from $(2.1)$

$$
\sigma(b)=\sigma\left(b_{0}\right) \sigma(h)=u^{-1} b=\left(u^{-1} b_{0}\right) h
$$

and thus, since $\sigma\left(\Lambda_{*}^{+} G^{\mathbb{C}}\right) \subseteq \Lambda_{*}^{+} G^{\mathbb{C}}$,

$$
\sigma\left(b_{0}\right)=u^{-1} b_{0}, \quad \sigma(h)=h .
$$

Now we express $b_{0} \in G^{\mathbb{C}}$ in its polar decomposition [15] $b_{0}=r \exp (i x)$, where $r \in G$ and $x \in \mathfrak{g}$. Applying $\sigma$ we get

$$
\sigma\left(b_{0}\right)=u^{-1} b_{0}=\sigma(r) \exp (i \sigma(x))=\left(u^{-1} r\right) \exp (i x)
$$

which implies

$$
\sigma(r)=u^{-1} r, \quad \exp (i \sigma(x))=\exp (i x) .
$$

Now let $K^{\mathbb{C}}=K \cdot B$ be the Iwasawa decomposition for $K^{\mathbb{C}}$ and decompose

$$
\exp (i x)=k v \in K \cdot B \text {. }
$$

Define

$$
\widetilde{a}=a r k, \quad \widetilde{b}=v h
$$

then, by (2.1), (2.2), (2.3) and the fact that $K^{\mathbb{C}}=\left(G^{\mathbb{C}}\right)^{\sigma}$,

$$
\begin{aligned}
& \sigma(\widetilde{a})=\sigma(a) \sigma(r) \sigma(k)=(a u)\left(u^{-1} r\right) k=a r k=\widetilde{a}, \\
& \sigma(\widetilde{b})=\sigma(v) \sigma(h)=v h=\widetilde{b},
\end{aligned}
$$


which shows that $\tilde{a} \in \Lambda G_{\sigma}$ and, since $v \in B, h \in \Lambda_{*}^{+} G^{\mathbb{C}}$, that $\widetilde{b} \in \Lambda_{B}^{+} G_{\sigma}^{\mathbb{C}}$. Moreover

$$
\widetilde{a} \widetilde{b}=(a r k)(v h)=a r \exp (i x) h=a\left(b_{0} h\right)=a b=g,
$$

which proves surjectivity of the multiplication map $\Lambda G_{\sigma} \times \Lambda_{B}^{+} G_{\sigma}^{\mathbb{C}} \rightarrow \Lambda G_{\sigma}^{\mathbb{C}}$.

(ii) This follows from Theorem 2.2.(ii) and the fact that

$$
\Lambda_{*}^{-} G_{\sigma}^{\mathbb{C}} \cdot \Lambda^{+} G_{\sigma}^{\mathbb{C}}=\left(\Lambda_{*}^{-} G^{\mathbb{C}} \cdot \Lambda^{+} G^{\mathbb{C}}\right) \cap \Lambda G_{\sigma}^{\mathbb{C}} .
$$

Another ingredient necessary for our studies of harmonic maps is the Grassmannian model for the loop group and its determinant line bundle [23], [26] which we review briefly in a language suitable for our developments. Since a compact semi-simple Lie group can be represented as a subgroup of the unitary group via the adjoint representation we begin with the loop groups $\Lambda \boldsymbol{U}(n)$ and its complexification $\Lambda \boldsymbol{G L}(n, \mathbb{C})$. Let $H=L^{2}\left(S^{1}, \mathbb{C}\right)$ with the "polarization"

$$
H=H_{+} \oplus H_{-},
$$

where $H_{+}$is the subspace generated by $\left\{\lambda^{k} ; k \geq 0\right\}$ and $H_{-}$is generated by $\left\{\lambda^{k} ; k<0\right\}$. Note that $\Lambda \boldsymbol{G L}(n, \mathbb{C})$ acts on $L^{2}\left(S^{1}, \mathbb{C}^{n}\right)$ by bounded linear operators so that, using an appropriate isometry between $L^{2}\left(S^{1}, \mathbb{C}^{n}\right)$ and $H$, we obtain an injective holomorphic group homomorphism

$$
\Lambda \boldsymbol{G L}(n, \mathbb{C}) \hookrightarrow G L(H): g \mapsto A_{g}
$$

of the loop group into the space of bounded linear operators on $H$. In terms of the natural basis $\ldots, \lambda^{2}, \lambda, 1, \lambda^{-1}, \lambda^{-2}, \ldots$ of $H_{+} \oplus H_{-}$this inclusion is expressed by infinite matrices: let

$$
g=\sum_{k \in \mathbb{Z}} g_{k} \lambda^{k}
$$

be the Fourier decomposition of $g \in \Lambda \boldsymbol{G L}(n, \mathbb{C})$, where $g_{k} \in M_{n}(\mathbb{C})$, then the $(i, j)$-th $n \times n$-block in $A_{g}$ is given by

$$
\left(A_{g}\right)_{i, j}=g_{i-j} .
$$

Writing elements $A \in G L(H)$ in block form with respect to the polarization $H=H_{+} \oplus H_{-}$,

$$
A=\left(\begin{array}{ll}
a & b \\
c & d
\end{array}\right)
$$


it follows from [23], [26] that the inclusion (2.5) takes values in

$$
G L_{r e s}(H)=\{A \in G L(H) ; \quad a, d \text { Fredholm , } \quad b, c \text { Hilbert-Schmidt }\} \text {. }
$$

The identity component of $\Lambda \mathbf{G} L(n, \mathbb{C})$ is mapped into the identity component of $G L_{r e s}(H)$ characterized by the condition that the Fredholm operators $a, d$ have index zero. Without further mentioning, we will work in these connected components. The Grassmannian of subspaces of $H$, comparable to $H_{+}$, is the orbit

$$
G r(H)=G L_{r e s}(H) \cdot H_{+},
$$

which is a complex homogeneous space on which $G L_{\text {res }}(H)$ acts holomorphically. One can make sense of the "top exterior power" of $W \in G r(H)$ [23], [26] and thus obtain a holomorphic line bundle

$$
\operatorname{Det} \rightarrow \operatorname{Gr}(H),
$$

the determinant line bundle. Its dual $\operatorname{Det}^{*} \rightarrow G r(H)$ has a non-trivial canonical holomorphic section

$$
\tau: G r(H) \rightarrow \operatorname{Det}^{*}
$$

given by

$$
\tau(W)=\left(w, \operatorname{det} w_{+}\right)
$$

where

$$
w=\left(\begin{array}{c}
w_{+} \\
w_{-}
\end{array}\right): H_{+} \rightarrow H
$$

is the $\mathbb{Z} \times \mathbb{N}$-matrix consisting of the columns of an admissible basis [26] for $W$. Consider the composition of holomorphic maps

$$
\Lambda \boldsymbol{G L}(n, \mathbb{C}) \hookrightarrow G L_{r e s}(H) \rightarrow G r(H): g \mapsto A_{g} \cdot H_{+}
$$

Lemma 2.4. $\tau\left(A_{g} \cdot H_{+}\right) \neq 0$ if and only if $g \in \Lambda \boldsymbol{G L}(n, \mathbb{C})$ is contained in the big cell (c.f. Theorem 2.2(ii)), i.e., $g=g_{-} g_{+}$with $g_{-} \in$ $\Lambda_{*}^{-} \boldsymbol{G L}(n, \mathbb{C}), g_{+} \in \Lambda^{+} \boldsymbol{G L}(n, \mathbb{C})$. 
Proof. From Birkhoff's factorization theorem [23] we know that

$$
g=g_{-} d g_{+}
$$

with $g_{-} \in \Lambda_{*}^{-} \boldsymbol{G L}(n, \mathbb{C})$ and $d=\operatorname{diag}\left(\lambda^{a_{1}}, \ldots, \lambda^{a_{n}}\right)$, where $a_{i} \in \mathbb{Z}$ satisfy $a_{1} \geq \cdots \geq a_{n}$ and $\sum_{i=1}^{n} a_{i}=0$. Then,

$$
\tau\left(A_{g} \cdot H_{+}\right)=\tau\left(A_{g_{-}} A_{d} A_{g_{+}} \cdot H_{+}\right)=\tau\left(A_{g_{-}} A_{d} \cdot H_{+}\right)
$$

since $A_{g_{+}} \cdot H_{+}=H_{+}$. Because $g_{-} \in \Lambda_{*}^{-} \boldsymbol{G L}(n, \mathbb{C})$ we have by $(2.6),(2.7)$

$$
A_{g_{-}}=\left(\begin{array}{cc}
a_{-} & 0 \\
b_{-} & c_{-}
\end{array}\right)
$$

where $a_{-}, c_{-}$are lower diagonal with $I_{n}$-blocks in the diagonal. Let $w=$ $\left(\begin{array}{c}w_{+} \\ w_{-}\end{array}\right)$be an admissible basis for $A_{d} \cdot H_{+}$. Then

$$
v=g_{-}\left(\begin{array}{c}
w_{+} \\
w_{-}
\end{array}\right) a_{-}=\left(\begin{array}{c}
a_{-} w_{+} a_{-}^{-1} \\
*
\end{array}\right)
$$

is an admissible basis [26] of $A_{g_{-}} A_{d} H_{+}$so that by (2.10)

$$
\tau\left(A_{g} \cdot H_{+}\right)=\left(v, \operatorname{det}\left(a_{-} w_{+} a_{-}^{-1}\right)\right)=\left(v, \operatorname{det} w_{+}\right) .
$$

Since $d=\lambda^{a_{1}} E_{a_{1}}+\cdots+\lambda^{a_{n}} E_{a_{n}}$, where $E_{a_{k}}=\operatorname{diag}(0, \ldots, 1, \ldots 0)$ with 1 in the $a_{k}$-th place, we get from $(2.6)$ that the $(i, j)$-th block in $A_{d}$ is given by

$$
\left(A_{d}\right)_{i, j}=\left\{\begin{array}{cl}
E_{a_{k}} & \text { for } \quad i-j=a_{k} \\
0 & \text { else }
\end{array}\right.
$$

Writing

$$
A_{d}=\left(\begin{array}{ll}
\alpha & \beta \\
\gamma & \delta
\end{array}\right)
$$

according to (2.7) we see that $\operatorname{det} w_{+} \neq 0$ if and only if $\alpha$ is invertible. Assuming $a_{n}<0$ we see that $\alpha$ is not invertible so that $\operatorname{det} w_{+}=0$. Conversely, if $d=I_{n}$, then $w_{+}=I d$ for the natural choice of $w$, and so $\operatorname{det} w_{+} \neq 0$.

If $G$ is a semisimple compact Lie group then $G \hookrightarrow U(n)$ and hence $G^{\mathbb{C}} \hookrightarrow$ $\boldsymbol{G L}(n, \mathbb{C})$, so that we obtain a holomorphic inclusion $\Lambda G_{\sigma}^{\mathbb{C}} \hookrightarrow \Lambda \boldsymbol{G L}(n, \mathbb{C})$.

Corollary 2.5. Let $g \in \Lambda G_{\sigma}^{\mathbb{C}}$. Then $g=g_{-} g_{+}$is in the big cell (c.f. Theorem 2.3(ii)) if and only if $\tau\left(A_{g} H_{+}\right) \neq 0$. 
As a further application, which will be needed in section 4, we show a certain meromorphic behavior in the factorization of a holomorphic $\Lambda G_{\sigma}^{\mathbb{C}}$ valued curve across the boundary of the big cell. Let $D \subset \mathbb{C}$ be some domain containing $0 \in \mathbb{C}$ and

$$
g: D \rightarrow \Lambda G_{\sigma}^{\mathbb{C}}
$$

a holomorphic (i.e., $\bar{\partial} g=0$ ) curve with $g(0)=e$.

Since $\tau: \Lambda G_{\sigma}^{\mathbb{C}} \rightarrow D e t^{*}$ and $g: D \rightarrow \Lambda G_{\sigma}^{\mathbb{C}}$ are holomorphic, we obtain from Corollary 2.5 that the set

$$
S=(\tau \circ g)^{-1}(0)=\{p \in D ; g(p) \text { not contained in the big cell }\} \subset D
$$

is discrete. Let $p_{0} \in S$ and $U \subset D$ be a neighborhood of $p_{0}$ so that (c.f. Theorem 2.3(ii))

$$
g(p)=g_{-}(p) g_{+}(p)
$$

for $p \in U \backslash\left\{p_{0}\right\}$ where $g_{ \pm}: U \backslash\left\{p_{0}\right\} \rightarrow \Lambda^{ \pm} G_{\sigma}^{\mathbb{C}}$ are holomorphic. Then we have

Lemma 2.6. $g_{-}$extends meromorphically into $p_{0}$, i.e., $g_{-}(\lambda): U \backslash\left\{p_{0}\right\} \rightarrow$ $G^{\mathbb{C}}$ has a pole of at most order $N$ independent of $\lambda \in S^{1}$ at $p_{0}$. Since $g$ is holomorphic the same statement holds for $g_{+}$.

Proof. Throughout the proof we will not distinguish between operators on $H=H_{-} \oplus H_{+}$and their representation as infinite matrices with respect to the standard basis $\ldots, \lambda^{2}, \lambda, 1, \lambda^{-1}, \lambda^{-2}, \ldots$ The map $g: D \rightarrow \Lambda G_{\sigma}^{\mathbb{C}}$ gives rise (2.9), (2.5) to a holomorphic map

$$
W=g \cdot H_{+}: D \rightarrow G r(H),
$$

which, on $U \backslash\left\{p_{0}\right\}$, is given by

$$
W=g_{-} \cdot H_{+} .
$$

If $g_{-}=e+\sum_{k \leq-1}\left(g_{-}\right)_{k} \lambda^{k}$ then (2.7), (2.6)

$$
A_{g_{-}}=\left(\begin{array}{cc}
a_{-} & 0 \\
c_{-} & d_{-}
\end{array}\right)
$$

with $a_{-}$lower diagonal and $\left(a_{-}\right)_{i i}=I_{n}$. In particular, $a_{-}: H_{+} \rightarrow H_{+}$is invertible. Thus,

$$
W(p)=\left[\begin{array}{l}
a_{-}(p) \\
c_{-}(p)
\end{array}\right]=\left[\begin{array}{l}
I d \\
c_{-}(p) a_{-}^{-1}(p)
\end{array}\right]
$$


where [] denotes the subspace generated by the columns. Using (2.6) and the special form of $a_{-}$we obtain

$$
\left(c_{-} a_{-}^{-1}\right)_{0, k}=\left(c_{-}\right)_{0, k}=\left(g_{-}\right)_{k}, \quad k \leq-1 .
$$

We now choose a chart around $W_{0}=A_{g\left(p_{0}\right)} \cdot H_{+}$: since by assumption $\tau\left(W_{0}\right)=0$, a chart is given by [9]

$$
\left\{\left[\begin{array}{c}
I d-y x \\
-x
\end{array}\right] ; x: H_{+} \rightarrow H_{-} \text {Hilbert-Schmidt }\right\}
$$

where $y: H_{-} \rightarrow H_{+}$is a "finite" matrix, i.e.,

$$
y=\left(\begin{array}{ll}
0 & 0 \\
f & 0
\end{array}\right)
$$

for some $f \in M_{m}(\mathbb{C}), m \in \mathbb{N}$. In this chart we express $W$ as

$$
\begin{aligned}
W(p)=A_{g_{-}(p)} \cdot H_{+} & =\left[\begin{array}{c}
I d-y x(p) \\
-x(p)
\end{array}\right] \\
& =\left[\begin{array}{c}
I d \\
-x(p)(I d-y x(p))^{-1}
\end{array}\right], \quad p \in U \backslash\left\{p_{0}\right\},
\end{aligned}
$$

where $x$ depends holomorphically on $p \in U$. Since $y$ is a finite matrix we have

$$
I d-y x(p)=\left(\begin{array}{c|c}
I d & 0 \\
\hline \alpha(p) & \beta(p)
\end{array}\right): H_{+} \rightarrow H_{+}
$$

with $\beta: U \rightarrow \boldsymbol{G L}(m, \mathbb{C})$ holomorphic and thus

$$
(I d-y x(p))^{-1}=\left(\begin{array}{c|c}
I d & 0 \\
\hline-\beta^{-1}(p) \alpha(p) & \beta^{-1}(p)
\end{array}\right) .
$$

Comparing (2.13) with (2.15) and using (2.14) we obtain

$$
\left(g_{-}\right)_{k}=\left(-x(I d-y x)^{-1}\right)_{0, k}, \quad k \leq-1,
$$

whose pole behavior at $p_{0}$ is determined by the behavior of $\beta^{-1}$ at $p_{0}$. But $\beta^{-1}: U \backslash\left\{p_{0}\right\} \rightarrow \boldsymbol{G L}(m, \mathbb{C})$ is holomorphic with pole at $p_{0}$ given by $\frac{1}{\operatorname{det} \beta}$. 


\section{Loop group formulation of harmonic maps into symmetric spaces.}

In this section we review what may be called the "Yang-Mills-Higgs" formulation of harmonic maps into a symmetric space [16, 7], which will lead to a loop group formulation similar to Uhlenbeck's [29] for harmonic maps into a Lie group.

Let $N=G / K$ be a Riemannian symmetric space with symmetric involution $\sigma: G \rightarrow G$ so that $G^{\sigma} \supset K \supset\left(G^{\sigma}\right)_{0}$. We denote by $\mathfrak{g}$ and $\mathfrak{k}$ the Lie algebras of $G$ and $K$ respectively. The decomposition of $\mathfrak{g}$ into \pm 1 eigenspaces of the derivative of $\sigma$ gives the Cartan decomposition

$$
\mathfrak{g}=\mathfrak{k} \oplus \mathfrak{p}
$$

with the familiar commutation relations $[\mathfrak{k}, \mathfrak{k}] \subseteq \mathfrak{k},[\mathfrak{k}, \mathfrak{p}] \subseteq \mathfrak{p}$ and $[\mathfrak{p}, \mathfrak{p}] \subseteq \mathfrak{k}$. Recall that there is a canonical identification of the tangent bundle $T N$ of $N$ with a subbundle of the trivial bundle $N \times \mathfrak{g}$ : for $x=g K \in N$ the map $\mathfrak{g} \rightarrow T_{x} N$ given by $\left.\eta \longmapsto \frac{d}{d t}\right|_{t=0} \exp (t \eta) x$ is a linear isomorphism on $\mathfrak{p}_{x}=\operatorname{Ad} g(\mathfrak{p})$. Inverting this at each point $x \in N$ gives a $\mathfrak{g}$-valued 1-form $\beta: T N \rightarrow \mathfrak{g}$ which identifies $T N$ with the adjoint bundle [p] $\subset N \times \mathfrak{g}$ whose fibre over $x=g K \in N$ is $\mathfrak{p}_{x}$. Under this identification the Levi-Civita connection of $N$ is just flat differentiation in $N \times \mathfrak{g}$ followed by projection along $[\mathfrak{k}]$ (defined in the same way as $[\mathfrak{p}]$ ) onto [p] , [8], i.e.,

$$
\beta\left(\stackrel{N}{\nabla}_{X} Y\right)=\left(d_{X} \beta(Y)\right)^{[\mathfrak{p}]} .
$$

Let $\pi: G \rightarrow G / K$ be the canonical projection and denote by $\theta: T G \rightarrow \mathfrak{g}$ the (left) Maurer-Cartan form of $G$. Then, according to (3.1),

$$
\theta=\theta_{0}+\theta_{1}
$$

decomposes into a $\mathfrak{k}$-valued 1 -form $\theta_{0}$ and a $\mathfrak{p}$-valued 1 -form $\theta_{1}$. $\theta_{0}$ is the canonical connection of the principal $K$-bundle $G \rightarrow G / K$ and $\theta_{1}$ is related to $\beta$ by

$$
\left(\pi^{*} \beta\right)_{g}=\operatorname{Ad} g\left(\theta_{1}\right)_{g} .
$$

Now let $M$ be a Riemann surface and $f: M \rightarrow G / K$ a smooth map with lift $F: M \rightarrow G$ so that $\pi \circ F=f$. Then the pullback

$$
\alpha=F^{-1} d F=F^{*} \theta=F^{*} \theta_{0}+F^{*} \theta_{1}=\alpha_{0}+\alpha_{1}
$$


splits into a $\mathfrak{k}$ resp. $\mathfrak{p}$-valued 1-form on $M$ and so does the Maurer-Cartan equation $d \alpha+1 / 2[\alpha \wedge \alpha]=0$ :

$$
\begin{aligned}
d \alpha_{0}+1 / 2\left[\alpha_{0} \wedge \alpha_{0}\right] & =-1 / 2\left[\alpha_{1} \wedge \alpha_{1}\right] \\
d \alpha_{1}+\left[\alpha_{0} \wedge \alpha_{1}\right] & =0 .
\end{aligned}
$$

Conversely, every pair of $\mathfrak{k}$ resp. $\mathfrak{p}$-valued 1 -forms $\alpha_{0}, \alpha_{1}$ solving (3.6) integrate, if $M$ is simply connected, to a map $F: M \rightarrow G$ which is unique up to left translation by $G$.

Recall that $f: M \rightarrow G / K=N$ is harmonic if and only if

$$
\nabla^{\prime \prime} \partial f=0,
$$

where $T M^{\mathbb{C}}=T^{\prime} M \oplus T^{\prime \prime} M$ is the decomposition into $(1,0)$ and $(0,1)$ tangent spaces, $d=\partial+\bar{\partial}$ and $\stackrel{N}{\nabla}=\nabla^{\prime}+\nabla^{\prime \prime}$. Using (3.2) this becomes

$$
\left(\bar{\partial} \beta^{\prime}\right)^{[\mathfrak{p}]}=0
$$

and thus, for a lift $F: M \rightarrow G$ of $f$, we obtain due to (3.4), (3.5)

$$
\left(\bar{\partial}\left(\operatorname{Ad} F \alpha_{1}^{\prime}\right)\right)^{[\mathfrak{p}]}=0
$$

which unravels to

$$
\bar{\partial} \alpha_{1}^{\prime}+\left[\alpha_{0}^{\prime \prime} \wedge \alpha_{1}^{\prime}\right]=\bar{\partial} \alpha_{1}^{\prime}+\left[\alpha_{0} \wedge \alpha_{1}^{\prime}\right]=0 .
$$

Since the sum of (3.10) and its complex conjugate give (3.6b) we arrive at the following characterization of a harmonic map $f: M \rightarrow G / K$ in terms of a lift $F: M \rightarrow G$.

Lemma 3.1. Let $f: M \rightarrow G / K$ be a smooth map with lift $F: M \rightarrow G$ and induced Maurer-Cartan form $\alpha=F^{-1} d F=\alpha_{0}+\alpha_{1}$. Then $f$ is harmonic iff

$$
\begin{aligned}
& d \alpha_{0}+1 / 2\left[\alpha_{0} \wedge \alpha_{0}\right]=-\left[\alpha_{1}^{\prime} \wedge \alpha_{1}^{\prime \prime}\right] \\
& \bar{\partial} \alpha_{1}^{\prime}+\left[\alpha_{0} \wedge \alpha_{1}^{\prime}\right]=0 .
\end{aligned}
$$

Conversely, if $\alpha_{0}: T M \rightarrow \mathfrak{k}$ and $\alpha_{1}: T M \rightarrow \mathfrak{p}$ are $\mathfrak{k}$-resp. $\mathfrak{p}$-valued 1-forms solving (3.11) and $M$ is simply connected, then there exists a unique (up to $G$-translation) harmonic map $f=\pi \circ F: M \rightarrow G / K$ where $F: M \rightarrow G$ integrates $F^{-1} d F=\alpha_{0}+\alpha_{1}$. 
Remark 3.2. Equations (3.11) are sometimes referred to as the YangMills-Higgs equations for the $K$-connection $\alpha_{0}$ and the Higgs-field $\alpha_{1}^{\prime}$. They are clearly invariant under the action of the Gauge group $C^{\infty}(M, K)$.

Similar to [29] we introduce a spectral parameter and associate to a harmonic map $f: M \rightarrow G / K$ an extended harmonic map into a certain loop space. Let $\alpha_{0}: T M \rightarrow \mathfrak{k}$ and $\alpha_{1}: T M \rightarrow \mathfrak{p}$ be $\mathfrak{k}$ resp. $\mathfrak{p}$-valued 1-forms on $M$. Then we have the following equivalent statements which follow immediately from a comparison of coefficients at like powers of $\lambda$ :

(i) $\alpha_{0}$ and $\alpha_{1}$ solve (3.11).

(ii) $\alpha_{0}$ and $\lambda^{-1} \alpha_{1}^{\prime}+\lambda \alpha_{1}^{\prime \prime}$ solve (3.11) for any $\lambda \in S^{1}$.

(iii) The $\Lambda \mathfrak{g}_{\sigma}$-valued 1-form

$$
A=\lambda^{-1} \alpha_{1}^{\prime}+\alpha_{0}+\lambda \alpha_{1}^{\prime \prime}
$$

solves the Maurer-Cartan equation $d A+\frac{1}{2}[A \wedge A]=0$.

In case $M$ is simply connected, (3.12 (iii)) can be integrated to a map

$$
\widetilde{F}: M \rightarrow \Lambda G_{\sigma}, \widetilde{F}^{-1} d \widetilde{F}=A
$$

and Lemma 3.1 and (3.12 (ii)) imply that

$$
f_{\lambda}=\pi \circ \widetilde{F}_{\lambda}: M \rightarrow G / K
$$

is harmonic for every $\lambda \in S^{1}$. Here $\widetilde{F}_{\lambda}$ denotes the composition $e v_{\lambda} \circ \widetilde{F}$ of $\widetilde{F}$ with the evaluation map $e v_{\lambda}: \Lambda G_{\sigma} \rightarrow G, e v_{\lambda}(g)=g(\lambda)$. This yields the following characterization of harmonic maps $f: M \rightarrow G / K$ when $M$ is simply connected.

Proposition 3.3.. Assume that $M$ is a simply connected Riemann surface. Then a map $f: M \rightarrow G / K$ is harmonic if and only if there exists a map $\widetilde{F}: M \rightarrow \Lambda G_{\sigma}$ with $\widetilde{F}^{-1} d \widetilde{F}=\lambda^{-1} \alpha_{1}^{\prime}+\alpha_{0}+\lambda \alpha_{1}^{\prime \prime}$ and $\pi \circ \widetilde{F}_{1}=f$, where $\alpha_{0}: T M \rightarrow \mathfrak{k}$ and $\alpha_{1}: T M \rightarrow \mathfrak{p}$ are $\mathfrak{k}$ resp. $\mathfrak{p}$-valued 1 -forms on $M$.

Definition 3.4. Let $f: M \rightarrow G / K$ be harmonic with $f\left(p_{0}\right)=e K$ and let $F: M \rightarrow G$ be a lift of $f$ with $F^{-1} d F=\alpha_{0}+\alpha_{1}$. Then $\widetilde{F}: M \rightarrow \Lambda G_{\sigma}$ integrating $\widetilde{F}^{-1} d \widetilde{F}=\lambda^{-1} \alpha_{1}^{\prime}+\alpha_{0}+\lambda \alpha_{1}^{\prime \prime}$ with initial condition $\widetilde{F}\left(p_{0}\right)=k \in K$ is called an extended lift of $f$. 
Remark 3.5. Given a harmonic map $f: M \rightarrow G / K$ an extended lift $\widetilde{F}: M \rightarrow \Lambda G_{\sigma}$ is determined only up to a gauge transformation $H: M \rightarrow K$, that is to say, $\widetilde{F} H: M \rightarrow \Lambda G_{\sigma}$ is also an extended lift. This freedom will be useful for the discussion in section 4. A gauge invariant description may be obtained by considering the homogeneous space $\Lambda G_{\sigma} / K$ which has a $\Lambda G_{\sigma^{-}}$ invariant complex structure given by the decomposition of the complexified tangent space at the base point $o=e K$

$$
T_{o}\left(\Lambda G_{\sigma} / K\right)^{\mathbb{C}}=\Lambda \mathfrak{g}_{\sigma}^{\mathbb{C}} / \mathfrak{k}^{\mathbb{C}}=\Lambda_{\sigma}^{-} \oplus \Lambda_{\sigma}^{+},
$$

where $\Lambda_{\sigma}^{\mp}=\left\{\xi \in \Lambda \mathfrak{g}_{\sigma}^{\mathbb{C}} \mid \xi=\sum_{k \lesseqgtr 0} \lambda^{k} \xi_{k}\right\}$. Further we call the finite dimensional $\Lambda G_{\sigma}$-invariant subbundle defined by $\Lambda G_{\sigma}$-translation of

$$
\left\{\lambda^{-1} \eta+\lambda \bar{\eta} \mid \eta \in \mathfrak{p}^{\mathbb{C}}\right\}
$$

the horizontal distribution. Then Proposition 3.3 gives the following familiar twistorial reformulation of the harmonicity condition.

Proposition 3.6.. Assume that $M$ is a simply connected Riemann surface. Then a map $f: M \rightarrow G / K$ is harmonic if and only if there exists a holomorphic and horizontal map $\tilde{f}: M \rightarrow \Lambda G_{\sigma} / K$ with $\widetilde{f}_{1}=f$.

Note, that unlike in Uhlenbeck's [29] setup for harmonic maps into Lie groups, $\widetilde{f}_{\lambda}: M \rightarrow G / K$ is harmonic for any $\lambda \in S^{1}$. Since any Lie group $G$ can be considered as the symmetric space $(G \times G) / \Delta G$ the present setup applies to this case as well. In analogy to the theory of minimal surfaces, $\widetilde{f}$ is usually called the associated family of harmonic maps to $f$.

\section{Weierstrass-type representation of harmonic maps.}

In this section we will discuss how every harmonic map $f: M \rightarrow G / K$ of a simply connected Riemann surface $M$ may be obtained from Weierstrasstype data. More specifically, we will show that $f$ can be reconstructed from a certain holomorphic 1-form with values in an infinite dimensional linear subspace of $\Lambda \mathfrak{g}_{\sigma}^{\mathbb{C}}$. Allowing certain singularities to occur we can do better and reconstruct $f$ from a $\mathfrak{p}^{\mathbb{C}}$-valued meromorphic 1-form. We also discuss how the finite type harmonic maps of $[5,7]$ arise from our point of view and finally apply our recipe to the construction of constant mean curvature surfaces with umbilic points.

Let $M$ be either $\mathbb{R}^{2}$ or the unit disk with global complex coordinate $z$. It will become clear below that our developments will not apply to $M=$ 
$S^{2}$ for which Uhlenbeck [29] already provided a complete description. It follows from Proposition 3.4, Definition 3.5 and Remark 3.6 that the space of harmonic maps $f: M \rightarrow G / K$ with $f(0)=e K$ can be identified with the space of extended lifts $\widetilde{F}: M \rightarrow \Lambda G_{\sigma}$ with $\widetilde{F}(0)=k \in K$ modulo gauge transformations $H: M \rightarrow K$ where $f=\pi \circ \widetilde{F}_{1}$.

Thus, if we denote the space of harmonic maps by

$$
\mathcal{H}=\{f: M \rightarrow G / K ; f(0)=e K, f \text { harmonic }\},
$$

we have a bijective correspondence

$$
\mathcal{H}=\left\{\widetilde{F}: M \rightarrow \Lambda G_{\sigma} ; \widetilde{F}(0)=k \in K, \widetilde{F} \text { extended lift }\right\} / \sim
$$

where $\widetilde{F}_{1} \sim \widetilde{F}_{2}$ if and only if $\widetilde{F}_{2}=\widetilde{F}_{1} H$ for a gauge $H: M \rightarrow K$. This identification will be used in the remainder of this section.

We now turn to the construction of harmonic maps in terms of holomorphic data. Recall from section 2, Theorem 2.3, that there is a global decomposition of the complexified $\sigma$-twisted loop group

$$
\Lambda G_{\sigma}^{\mathbb{C}}=\Lambda G_{\sigma} \cdot \Lambda_{B}^{+} G_{\sigma}^{\mathbb{C}}
$$

where $K^{\mathbb{C}}=K B$ is an Iwasawa decomposition for $K^{\mathbb{C}}$. Correspondingly, the Lie algebra $\Lambda \mathfrak{g}_{\sigma}^{\mathbb{C}}$ splits into a direct sum of Lie subalgebras

$$
\Lambda \mathfrak{g}_{\sigma}^{\mathbb{C}}=\Lambda \mathfrak{g}_{\sigma} \oplus \Lambda_{\mathfrak{b}}^{+} \mathfrak{g}_{\sigma}^{\mathbb{C}} .
$$

We will need the following linear subspace of $\Lambda \mathfrak{g}_{\sigma}^{\mathbb{C}}$ : for $d \in \mathbb{N}$ we let

$$
\Lambda_{d}=\left\{\xi \in \Lambda \mathfrak{g}_{\sigma} \mid \xi=\sum_{|k| \leq d} \lambda^{k} \xi_{k}\right\}
$$

be the finite dimensional subspace of Laurant polynomial loops of degree not larger than $d$. Further we introduce the space of $\sigma$-twisted loops which extend holomorphically to the interior of the $\lambda$-disc and have a simple pole at the origin

$$
\Lambda_{-1, \infty}:=\left\{\xi \in \Lambda \mathfrak{g}_{\sigma}^{\mathbb{C}} \mid \lambda \xi \text { extends holomorphically to }|\lambda|<1\right\} .
$$


As a Fourier series, $\xi \in \Lambda_{-1, \infty}$ if and only if

$$
\xi=\sum_{k \geq-1} \lambda^{k} \xi_{k}, \quad \xi_{\text {even }} \in \mathfrak{k}^{\mathbb{C}}, \quad \xi_{\text {odd }} \in \mathfrak{p}^{\mathbb{C}} .
$$

Notice that $\Lambda_{-1, \infty}$ is a closed subspace of $\Lambda \mathfrak{g}_{\sigma}^{\mathbb{C}}$ which is stable under the adjoint action of $\Lambda^{+} G_{\sigma}^{\mathbb{C}}$ and so in particular under $\Lambda_{B}^{+} G_{\sigma}^{\mathbb{C}}$.

Definition 4.1. The vector space $\mathcal{P}$ of holomorphic 1-forms on $M$ with values in the Banach space $\Lambda_{-1, \infty}$ will be called the space of holomorphic potentials. Thus $\mu \in \mathcal{P}$ is a holomorphic potential if $\mu=\sum_{k \geq-1} \lambda^{k} \mu_{k}$, where $\mu_{\text {even }}$ resp. $\mu_{\text {odd }}$ are holomorphic $\mathfrak{k}^{\mathbb{C}}$ resp. $\mathfrak{p}^{\mathbb{C}}$-valued 1-forms on $M$.

The construction outlined below shows why the space $\mathcal{P}$ serves as the space of "Weierstrass-data" for the construction of harmonic maps: if $\mu \in \mathcal{P}$ is a holomorphic potential, then

$$
\mu=\xi d z
$$

for some holomorphic function $\xi=\sum_{k \geq-1} \lambda^{k} \xi_{k}: M \rightarrow \Lambda_{-1, \infty}$. Thus

$$
d \mu+\frac{1}{2}[\mu \wedge \mu]=\bar{\partial} \mu=0,
$$

and we can integrate

$$
g_{\mu}^{-1} d g_{\mu}=\mu, g_{\mu}(0)=e,
$$

to obtain a unique map

$$
g_{\mu}: M \rightarrow \Lambda G_{\sigma}^{\mathbb{C}} .
$$

Decomposing $g_{\mu}$ according to (4.3),

$$
g_{\mu}=\Phi_{\mu} b_{\mu},
$$

yields a map $\Phi_{\mu}: M \rightarrow \Lambda G_{\sigma}$ with $\Phi_{\mu}(0)=e$.

Lemma 4.2. $\Phi_{\mu}: M \rightarrow \Lambda G_{\sigma}$ is an extended harmonic lift.

Proof. According to Proposition 3.3 we have to verify that

$$
\Phi_{\mu}^{-1} d \Phi_{\mu}=\lambda^{-1} \alpha_{1}^{\prime}+\alpha_{0}+\lambda \alpha_{1}^{\prime \prime} .
$$


Since $\Phi_{\mu}=g b^{-1}$ (surpressing the subscript $\mu$ in $g$ and $b$ ) we have

$$
\begin{aligned}
\Phi_{\mu}^{-1} d \Phi_{\mu} & =\operatorname{Ad} b\left(g^{-1} d g\right)-d b b^{-1} \\
& =\operatorname{Ad} b(\mu)-d b b^{-1} .
\end{aligned}
$$

But $b$ takes values in $\Lambda_{B}^{+} G_{\sigma}^{\mathbb{C}}$, so that $d b b^{-1}$ takes values in $\Lambda_{\mathfrak{b}}^{+} \mathfrak{g}_{\sigma}^{\mathbb{C}}$, hence by

$$
\Phi_{\mu}^{-1} d \Phi_{\mu}=(\operatorname{Ad} b(\mu))_{\Lambda \mathfrak{g}_{\sigma}} .
$$

Since $\mu=\sum_{k \geq-1} \mu_{k} \lambda^{k}$ is $\Lambda_{-1, \infty}$-valued and $\operatorname{Ad} \Lambda_{B}^{+} G_{\sigma}^{\mathbb{C}}$ acts on $\Lambda_{-1, \infty}$ we obtain, by putting $b_{\left.\right|_{\lambda=0}}=b_{0}: M \rightarrow B$,

$$
(\operatorname{Ad} b(\mu))_{\Lambda \mathfrak{g} \sigma}=\lambda^{-1} \operatorname{Ad} b_{0}\left(\mu_{-1}\right)+\alpha_{0}+\lambda \operatorname{Ad} \overline{b_{0}}\left(\overline{\mu_{-1}}\right)
$$

for some $\mathfrak{k}$-valued 1 -form $\alpha_{0}: T M \rightarrow \mathfrak{k}$. Finally note that $\mu_{-1}=\xi_{-1} d z$ is a $\mathfrak{p}^{\mathbb{C}}$-valued holomorphic differential and thus $\operatorname{Ad} b_{0}\left(\mu_{-1}\right)$ is a 1-form of type $(1,0)$.

If we recall the identification made in (4.2), the above proposition provides us with a map

$$
\Phi: \mathcal{P} \longrightarrow \mathcal{H}: \mu \longmapsto\left[\Phi_{\mu}\right]
$$

which we think of as a Weierstrass-type representation for harmonic maps.

Before we investigate the properties of this map, let us pause and discuss how this construction relates to the finite type harmonic maps in [5]. It is shown in [7] that a harmonic map $f: \mathbb{R}^{2} \rightarrow G / K$ is of finite type if $f$ is obtained from an extended lift $\Phi_{\mu}: \mathbb{R}^{2} \rightarrow \Lambda G_{\sigma}$ whose holomorphic potential $\mu=\stackrel{\circ}{\xi} d z$ is constant of the form

$$
\stackrel{\circ}{\xi}=\lambda^{d-1} \stackrel{\circ}{\eta}, \quad \stackrel{\circ}{\eta} \in \Lambda_{d},
$$

for some $d \in \mathbb{N}$ odd. Otherwise said, for $d \in \mathbb{N}$ odd, we have natural subspaces

$$
\lambda^{d-1} \Lambda_{d} d z \subset \mathcal{P},
$$

and the images of those subspaces under $\Phi$ comprise the finite type harmonic maps. The importance of finite type harmonic maps lies in the fact that they can be constructed by solving finite dimensional Hamiltonian ODE's 
in Lax form. We briefly sketch these developments: let $d \in \mathbb{N}$ be odd,

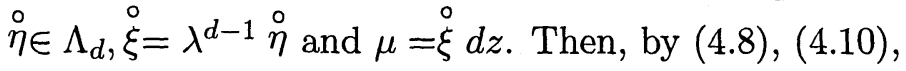

$$
g_{\mu}(z)=\exp \left(\lambda^{d-1} \stackrel{\circ}{\eta} z\right)=\Phi_{\mu}(z) b_{\mu}(z)
$$

Let

$$
\eta:=\operatorname{Ad} \Phi_{\mu}^{-1}(\stackrel{\circ}{\eta}): M \rightarrow \Lambda \mathfrak{g}_{\sigma}
$$

Then, by (4.17),

$$
\eta=\operatorname{Ad} b_{\mu}(\stackrel{\circ}{\eta})
$$

and thus

$$
\lambda^{d} \eta=\operatorname{Ad} b_{\mu}\left(\lambda^{d} \stackrel{\circ}{\eta}\right)
$$

which, since $b_{\mu}$ takes values in $\Lambda_{B}^{+} G_{\sigma}^{\mathbb{C}}$, extends holomorphically to $|\lambda|<1$. Hence $\eta$ has a pole of order $d$ at $\lambda=0$ and is real due to (4.18), so

$$
\eta: M \rightarrow \Lambda_{d} \subset \Lambda \mathfrak{g}_{\sigma}
$$

is $\Lambda_{d}$-valued. Moreover,

$$
d \eta=\left[\eta, \Phi_{\mu}^{-1} d \Phi_{\mu}\right]
$$

and from (4.19), (4.12) we obtain

$$
\Phi_{\mu}^{-1} d \Phi_{\mu}=\left(\operatorname{Ad} b_{\mu}(\mu)\right)_{\Lambda \mathfrak{g} \sigma}=\left(\operatorname{Ad} b_{\mu}\left(\lambda^{d-1} \stackrel{\circ}{\eta} d z\right)\right)_{\Lambda_{\mathfrak{g} \sigma}}=\left(\lambda^{d-1} \eta\right)_{\Lambda \mathfrak{g}_{\sigma}} .
$$

Combining (4.21) and (4.22) we see that the $\Lambda_{d}$-valued map $\eta$ satisfies the following system of ODE's in Lax-form:

$$
d \eta=\left[\eta,\left(\lambda^{d-1} \eta d z\right)_{\Lambda \mathfrak{g}_{\sigma}}\right], \quad \eta(0)=\stackrel{\circ}{\eta} \in \Lambda_{d} .
$$

Readers familiar with the Adler-Kostant-Symes integration scheme [1] will recognize (4.23) as a special case of commuting Hamiltonian flows on $\Lambda \mathfrak{g}_{\sigma}$ (cf.[7]). From this theory it follows that (4.23) has a unique, globally defined solution

$$
\eta: M \rightarrow \Lambda_{d}
$$

to any choice of initial condition $\eta_{0} \in \Lambda_{d}$. (That $\eta$ is globally defined follows from the fact that in our case the $L^{2}$-inner product on $\Lambda \mathfrak{g}_{\sigma}$ is positive definite 
and is preserved by the flows (4.23) which evolve on the finite dimensional subspace $\Lambda_{d}$.) Moreover, when $d \in \mathbb{N}$ is odd,

$$
A=\left(\lambda^{d-1} \eta d z\right)_{\Lambda \mathfrak{g}_{\sigma}}: T M \rightarrow \Lambda \mathfrak{g}_{\sigma}
$$

is of the form

$$
A=\lambda^{-1} \alpha_{1}^{\prime}+\alpha_{0}+\lambda \alpha_{1}^{\prime \prime}
$$

satisfies the Maurer-Cartan equation $d A+\frac{1}{2}[A, A]=0$ and can thus be integrated to a map

$$
\Phi: M \rightarrow \Lambda G_{\sigma}, \Phi(0)=e .
$$

Clearly, $\Phi=\Phi_{\mu}$ with $\mu=\lambda^{d-1} \stackrel{\circ}{\eta} d z$, so that by solving (4.23) one finds all extended lifts $\Phi_{\mu}$ of finite type harmonic maps $f: M \rightarrow G / K$. For a more detailed description of this and also its relation to the $r$-matrix integration scheme see [7].

After this excursion into finite type harmonic maps, we return to the Weierstrass representation $\Phi: \mathcal{P} \rightarrow \mathcal{H}(4.14)$. First we observe that $\Phi$ is equivariant with respect to natural group actions on $\mathcal{P}$ and $\mathcal{H}$. The group decomposition (4.3)

$$
\Lambda G_{\sigma}^{\mathbb{C}}=\Lambda G_{\sigma} \cdot \Lambda_{B}^{+} G_{\sigma}^{\mathbb{C}}
$$

defines a "dressing action" [32] of $\Lambda G_{\sigma}^{\mathbb{C}}$ on $\Lambda G_{\sigma}$ : for $h \in \Lambda G_{\sigma}^{\mathbb{C}}$ and $g \in \Lambda G_{\sigma}$

$$
h \# g:=(h g)_{\Lambda G_{\sigma}} .
$$

In particular, $\Lambda^{+} G_{\sigma}^{\mathbb{C}}$ acts via dressing on $\Lambda G_{\sigma}$. Now let $F: M \rightarrow \Lambda G_{\sigma}$ be an extended lift and $h \in \Lambda^{+} G_{\sigma}^{\mathbb{C}}$. Applying (4.26) pointwise, we obtain a new map

$$
h \# F: M \rightarrow \Lambda G_{\sigma}, \quad(h \# F)(z)=h \# F(z), \quad z \in M .
$$

Similar to the case of harmonic maps into Lie groups [14] this defines an action on $\mathcal{H}$.

Proposition 4.3. $\quad$ (i) $h \# F$ is an extended lift.

(ii) Let $H: M \rightarrow K$ be a gauge transformation. Then there exists $\widetilde{H}: M \rightarrow$ $K$ with

$$
h \#(F H)=(h \# F) \tilde{H},
$$


i.e., $h \#(F H)$ and $h \# F$ are extended lifts of the same harmonic map. Thus (4.27) defines a left action of $\Lambda^{+} G_{\sigma}^{\mathbb{C}}$ on $\mathcal{H}$ given by

$$
h \# f=\pi \circ\left((h \# F)_{\left.\right|_{\lambda=1}}\right)
$$

where $F: M \rightarrow \Lambda G_{\sigma}$ is an extended lift of the harmonic map $f: M \rightarrow$ $G / K$.

Proof. (i) This computation is analogous to the one given in [14]. Since $F$ is an extended lift there exists $\mathfrak{k}$ and $\mathfrak{p}$-valued 1 -forms $\alpha_{0}, \alpha_{1}$ on $M$ with

$$
F^{-1} d F=\lambda^{-1} \alpha_{1}^{\prime}+\alpha_{0}+\lambda \alpha_{1}^{\prime \prime} .
$$

Now let $h \in \Lambda^{+} G_{\sigma}^{\mathbb{C}}$ and decompose

$$
h F=(h \# F) b
$$

according to (4.3) where $b: M \rightarrow \Lambda_{B}^{+} G_{\sigma}^{\mathbb{C}}$. Then

$$
\begin{aligned}
(h \# F)^{-1} d(h \# F) & =b F^{-1} h^{-1}\left(h d F b^{-1}-h F b^{-1} d b b^{-1}\right) \\
& =\operatorname{Ad} b\left(F^{-1} d F\right)-d b b^{-1}
\end{aligned}
$$

and since $d b b^{-1}$ takes values in $\Lambda_{\mathfrak{b}}^{+} \mathfrak{g}_{\sigma}^{\mathbb{C}}$ we obtain

$$
(h \# F)^{-1} d(h \# F)=\operatorname{Ad} b\left(F^{-1} d F\right)_{\Lambda \mathfrak{g}_{\sigma}} .
$$

Recall that $\operatorname{Ad} \Lambda_{B}^{+} G_{\sigma}^{\mathbb{C}}$ preserves $\Lambda_{-1, \infty}$ so that, putting $b_{\left.\right|_{\lambda=0}}=b_{0} \in B \subset K^{\mathbb{C}}$,

$$
\left(\lambda \operatorname{Ad} b\left(F^{-1} d F\right)\right)_{\left.\right|_{\lambda=0}}=\operatorname{Ad} b_{0}\left(\alpha_{1}^{\prime}\right)
$$

and thus

$$
\left(\operatorname{Adb}\left(F^{-1} d F\right)\right)_{\Lambda_{\sigma}}=\lambda^{-1} \widetilde{\alpha}_{1}^{\prime}+\widetilde{\alpha}_{0}+\lambda \widetilde{\alpha}_{1}^{\prime \prime}
$$

where the $\mathfrak{p}$-valued 1 -form $\widetilde{\alpha}_{1}=\widetilde{\alpha}_{1}^{\prime}+\widetilde{\alpha}_{1}^{\prime \prime}$ is given by

$$
\widetilde{\alpha}_{1}^{\prime}=\operatorname{Ad} b_{0}\left(\alpha_{1}^{\prime}\right)
$$

and $\widetilde{\alpha}_{0}$ is some $\mathfrak{k}$-valued 1-form. Moreover, since $F(0)=k \in K$ and $\Lambda G_{\sigma} \cap$ $\Lambda^{+} G_{\sigma}^{\mathbb{C}}=K$, we get

$$
(h \# F)(0)=h \# F(0)=h \# k=(h k)_{\Lambda G_{\sigma}} \in K .
$$

Hence $h \# F$ is an extended lift. 
(ii) Let $H: M \rightarrow K$ and $h \in \Lambda^{+} G_{\sigma}^{\mathbb{C}}$. Then

$$
\begin{aligned}
h F & =(h \# F) b \\
h F H & =(h \#(F H)) \widetilde{b}=(h \# F) b H
\end{aligned}
$$

for $b, \tilde{b}: M \rightarrow \Lambda_{B}^{+} G_{\sigma}^{\mathbb{C}}$. Thus

$$
h \#(F H)=(h \# F) b H \widetilde{b}^{-1}
$$

and we conclude that $b H \tilde{b}^{-1}$ takes values in $\Lambda G_{\sigma}$. But $b H \tilde{b}^{-1}$ also takes values in $\Lambda^{+} G_{\sigma}^{\mathbb{C}}$ so that

$$
b H \widetilde{b}^{-1}=: \widetilde{H}
$$

takes values in $K$.

The space $\mathcal{P}$ of holomorphic potentials admits a natural left action of the holomorphic gauge group

$$
\mathcal{G}=\left\{h: M \rightarrow \Lambda^{+} G_{\sigma}^{\mathbb{C}} ; \bar{\partial} h=0\right\}
$$

by gauge transformations, i.e., for $\mu \in \mathcal{P}$ and $h \in \mathcal{G}$

$$
h \cdot \mu=\operatorname{Ad} h(\mu)-d h h^{-1} .
$$

Lemma 4.4. The map $\Phi: \mathcal{P} \rightarrow \mathcal{H}: \mu \rightarrow\left[\Phi_{\mu}\right]$ is equivariant with respect to the gauge action of $\mathcal{G}$ on $\mathcal{P}$ and the dressing action of $\Lambda^{+} G_{\sigma}^{\mathbb{C}}$ on $\mathcal{H}$. More specifically, for $h \in \mathcal{G}$

$$
\left[\Phi_{h \cdot \mu}\right]=h(0) \#\left[\Phi_{\mu}\right] .
$$

Proof. We have to show that for some $H: M \rightarrow K$

$$
\Phi_{h \cdot \mu}=\left(h(0) \# \Phi_{\mu}\right) H .
$$

From (4.8) and (4.10) we see that $\Phi_{\mu}=\left(g_{\mu}\right)_{\Lambda G_{\sigma}}$ where $g_{\mu}: M \rightarrow \Lambda G_{\sigma}^{\mathbb{C}}$ solves $g^{-1} d g=\mu$ subject to $g(0)=e$. Since $h(0) g_{\mu} h^{-1}$ solves $g^{-1} d g=h \cdot \mu$ subject to $g(0)=e$ we conclude

$$
g_{h \cdot \mu}=h(0) g_{\mu} h^{-1} .
$$

Let $b_{\mu}: M \rightarrow \Lambda_{B}^{+} G_{\sigma}^{\mathbb{C}}$ such that $g_{\mu}=\Phi_{\mu} b_{\mu}$. Since $\Lambda^{+} G_{\sigma}^{\mathbb{C}}=K \cdot \Lambda_{B}^{+} G_{\sigma}^{\mathbb{C}}$ and $h$ takes values in $\Lambda^{+} G_{\sigma}^{\mathbb{C}}$ we have

$$
\begin{aligned}
\Phi_{h \cdot \mu} & =\left(g_{h \cdot \mu}\right)_{\Lambda G_{\sigma}}=\left(h(0) g_{\mu} h^{-1}\right)_{\Lambda G_{\sigma}} \\
& =\left(h(0) \Phi_{\mu} b_{\mu} h^{-1}\right)_{\Lambda G_{\sigma}}=\left(h(0) \Phi_{\mu} \widetilde{H}\right)_{\Lambda G_{\sigma}}
\end{aligned}
$$


where $b_{\mu} h^{-1}=\widetilde{H} \tilde{b}$ with $\widetilde{H}: M \rightarrow K$ and $\widetilde{b}: M \rightarrow \Lambda_{B}^{+} G_{\sigma}^{\mathbb{C}}$. But by (4.27), the latter is $h(0) \#\left(\Phi_{\mu} \widetilde{H}\right)$ and so proposition 4.3 (ii) gives

$$
\Phi_{h \cdot \mu}=h(0) \#\left(\Phi_{\mu} \widetilde{H}\right)=\left(h(0) \# \Phi_{\mu}\right) H
$$

for some $H: M \rightarrow K$.

A crucial property of our construction is the fact that every harmonic map can be obtained from some holomorphic potential. This reduces the harmonic map equation to a linear ODE with holomorphic data similar to the classical Weierstrass representation for minimal surfaces.

Lemma 4.5. The map $\Phi: \mathcal{P} \rightarrow \mathcal{H}: \mu \rightarrow\left[\Phi_{\mu}\right]$ is surjective and its fibres are the orbits of the based holomorphic gauge group $\mathcal{G}_{0}=\{h \in \mathcal{G} ; h(0)=e\}$.

Proof. Let $f: M \rightarrow G / K$ be harmonic and let $F: M \rightarrow \Lambda G_{\sigma}$ be an extended lift of $f$ which we may assume has $F(0)=e$. We have to find a holomorphic potential $\mu \in \mathcal{P}$ with $\left[\Phi_{\mu}\right]=[F]$. The idea is to construct $h: M \rightarrow \Lambda^{+} G_{\sigma}^{\mathbb{C}}$ with $h(0)=e$ so that $g=F h$ has $g_{\bar{z}}=0$, or equivalently, that $\mu=g^{-1} d g$ is a 1-form of type $(1,0)$ which, due to the Maurer-Cartan equation, is then holomorphic. Since $F^{-1} d F=\lambda^{-1} \alpha_{1}^{\prime}+\alpha_{0}+\lambda \alpha_{1}^{\prime \prime}$ the holomorphic 1-form $\mu$ takes values in $\Lambda_{-1, \infty}$, i.e., $\mu \in \mathcal{P}$. Moreover, due to $\Lambda^{+} G_{\sigma}^{\mathbb{C}}=K \cdot \Lambda_{B}^{+} G_{\sigma}^{\mathbb{C}}$, we have $h=H b$ with $H: M \rightarrow K$ and $b: M \rightarrow \Lambda_{B}^{+} G_{\sigma}^{\mathbb{C}}$ so that

$$
g=(F H) b .
$$

Comparing with (4.10) and noting that $g(0)=e$ we see that

$$
\Phi_{\mu}=F H
$$

and thus $\left[\Phi_{\mu}\right]=[F]$. As outlined above, we want to find $h: M \rightarrow \Lambda^{+} G_{\sigma}^{\mathbb{C}}$ such that the $(0,1)$-part of $\mu$ vanishes, i.e., we need to solve the equation

$$
\mu^{(0,1)}=0 \text {. }
$$

But

$$
\begin{aligned}
g^{-1} d g & =\operatorname{Ad} h^{-1}\left(F^{-1} d F\right)+h^{-1} d h \\
& =\operatorname{Ad} h^{-1}\left(\lambda^{-1} \alpha_{1}^{\prime}+\alpha_{0}+\lambda \alpha_{1}^{\prime \prime}\right)+h^{-1} d h
\end{aligned}
$$

so that (4.31) becomes

$$
\bar{\partial} h h^{-1}=-\left(\alpha_{0}^{\prime \prime}+\lambda \alpha_{1}^{\prime \prime}\right)
$$


subject to $h(0)=e$. This is a $\bar{\partial}$-problem with right hand side in the Banach Lie algebra $\Lambda^{+} \mathfrak{g}_{\sigma}^{\mathbb{C}}$ which can be solved over the simply connected domain $M$ (see Appendix). Note that solutions of (4.32) subject to $h(0)=e$ are determined only up to right multiplication by elements in $\mathcal{G}_{0}$. So let $h: M \rightarrow$ $\Lambda^{+} G_{\sigma}^{\mathbb{C}}$ be a solution of (4.32) with $h(0)=e$. Then, by construction, $g=F h$ has

$$
\mu=g^{-1} d g=\operatorname{Ad} h^{-1}\left(\lambda^{-1} \alpha_{1}^{\prime}+\alpha_{0}^{\prime}\right)+h^{-1} \partial h
$$

which is of type $(1,0)$ and thus holomorphic. Moreover, since $h$ takes values in $\Lambda^{+} G_{\sigma}^{\mathbb{C}}$ and $\operatorname{Ad} \Lambda^{+} G_{\sigma}^{\mathbb{C}}$ leaves $\Lambda_{-1, \infty}$ invariant, $\mu$ takes values in $\Lambda_{-1, \infty}$ which is to say $\mu \in \mathcal{P}$. Thus $\Phi: \mathcal{P} \rightarrow \mathcal{H}$ is surjective. To determine the fibres of $\Phi$, let $\mu, \eta \in \mathcal{P}$ such that

$$
\left[\Phi_{\mu}\right]=\left[\Phi_{\eta}\right] .
$$

Then $\Phi_{\mu}=\Phi_{\eta} H$ with $H: M \rightarrow K$ and since by (4.10) $g_{\mu}=\Phi_{\mu} b_{\mu}, g_{\eta}=\Phi_{\eta} b_{\eta}$ we see that (4.33) is equivalent with

$$
g_{\mu}=g_{\eta}\left(b_{\eta}^{-1} H b_{\mu}\right)=g_{\eta} h^{-1}
$$

where $h: M \rightarrow \Lambda^{+} G_{\sigma}^{\mathbb{C}}$ with $h(0)=e$. Thus

$$
\mu=g_{\mu}^{-1} d g_{\mu}=\operatorname{Ad} h\left(g_{\eta}^{-1} d g_{\eta}\right)-d h h^{-1}=h \cdot \eta
$$

and from (4.34) we see that $h \in \mathcal{G}_{0}$. Thus $\mu$ and $\eta$ are gauge equivalent under $\mathcal{G}_{0}$. The converse is obvious from Lemma 4.4.

Corollary 4.6. Every harmonic map $f: M \rightarrow G / K$ is obtained from a holomorphic potential $\mu \in \mathcal{P}$ such that $\mu=\sum_{k o d d, k \geq-1} \lambda^{k} \mu_{k}$, i.e., $\mu$ has only odd terms in $\lambda$, in particular, $\mu$ has no constant term.

Proof. This follows at once from the previous lemma and the fact that every $\eta \in \mathcal{P}$ is $\mathcal{G}_{0}$-equivalent to a $\mu \in \mathcal{P}$ with no even terms: let $\eta \in \mathcal{P}$ and decompose

$$
\eta=\eta_{e}+\eta_{o}
$$

into even and odd terms, i.e., $\eta_{e}(-\lambda)=\eta_{e}(\lambda)$ and $\eta_{o}(-\lambda)=-\eta_{o}(\lambda)$. In particular, $\eta_{e}$ takes values in $\Lambda^{+} \mathcal{G}_{\sigma}^{\mathbb{C}}$, so that there is a unique solution $h \in \mathcal{G}_{0}$ solving

$$
h^{-1} \partial h=\eta_{e}, \quad h(0)=e
$$

and, as a consequence,

$$
h(-\lambda)=h(\lambda) .
$$


Thus

$$
\begin{aligned}
\mu:=h \cdot \eta & =\operatorname{Ad} h(\eta)-d h h^{-1} \\
& =\operatorname{Ad} h\left(\eta_{e}\right)-d h h^{-1}+\operatorname{Ad} h\left(\eta_{0}\right)=\operatorname{Ad} h\left(\eta_{0}\right),
\end{aligned}
$$

where

$$
\mu(-\lambda)=\operatorname{Ad} h(-\lambda) \eta_{o}(-\lambda)=-\mu(\lambda)
$$

so that

$$
\mu=\sum_{k o d d, k \geq-1} \lambda^{k} \mu_{k}
$$

We summarize the above results in the following:

Theorem 4.7. Let $\Phi: \mathcal{P} \rightarrow \mathcal{H}$ be the Weierstrass representation of harmonic maps from an open simply connected Riemann surface into a symmetric space. Then

(i) $\Phi$ is equivariant, i.e., if $h \in \mathcal{G}$ then

$$
\left[\Phi_{h \cdot \mu}\right]=h(0) \#\left[\Phi_{\mu}\right]
$$

(ii) $\Phi$ is surjective and

(iii) the fibres of $\Phi$ are the $\mathcal{G}_{0}$-gauge orbits in $\mathcal{P}$.

Remark 4.8. The above equivariance statement is the starting point for an investigation of the harmonic map equation along the lines of Segal and Wilson's [26] approach to the $K d V$-equation. The basic idea is that a sufficiently interesting class of solutions to the equations at hand is comprised by the dressing orbit through a "vacuum" solution. In the case of harmonic maps $f: M \rightarrow G / K$ into a symmetric space "vacuum" solutions are orbits of 2-parameter subgroups

$$
f_{C}(z)=\exp (C z+\bar{C} \bar{z}) \cdot o
$$

where $C \in \mathfrak{p}^{\mathbb{C}}$ with $[C, \bar{C}]=0$ and $o=e K$. An extended lift is easily seen to be

$$
F_{C}(z)=\exp \left(\lambda^{-1} C z+\lambda \bar{C} \bar{z}\right) .
$$

Since

$$
\exp \left(\lambda^{-1} C z\right)=F_{C} \cdot \exp (-\lambda \bar{C} \bar{z})
$$


and $\exp (-\lambda \bar{C} \bar{z})$ takes values in $\Lambda_{B}^{+} G_{\sigma}^{\mathbb{C}}$ we obtain by comparison to (4.10) that

$$
F_{C}=\Phi_{\mu}
$$

with the holomorphic potential $\mu=\lambda^{-1} C d z$. Recall that a harmonic map is of finite type (4.15) if it is of the form $\left[\Phi_{\mu}\right]$ with $\mu=\lambda^{d-1} \stackrel{\circ}{\eta}, \stackrel{\circ}{\eta} \in \Lambda_{d}, d \in \mathbb{N}$ odd. It has been shown in [5], [7] that every harmonic 2-torus $f: T^{2} \rightarrow G / K$ whose derivative $\frac{\partial f}{\partial z}: T^{2} \rightarrow T(G / K)^{\mathbb{C}}$ is regular semisimple at one point is of finite type. It is interesting to note that all such harmonic 2-tori are in fact contained in the dressing orbit of some $f_{C}$. Without going too much into details (one has to enlargen the loop groups involved slightly) we have the following more general result which is proven in [6]:

Theorem 4.9. Let $\mu=\sum_{k \geq-1} \xi_{k} \lambda^{k} d z \in \Lambda_{-1, \infty}$ be a constant (holomorphic) potential, i.e., $\xi_{\text {even }} \in \mathfrak{k}^{\mathbb{C}}, \xi_{\text {odd }} \in \mathfrak{p}^{\mathbb{C}}$, such that $\xi_{-1} \in \mathfrak{p}^{\mathbb{C}}$ is semisimple. Then there exists $h \in \Lambda^{+} G_{\sigma}^{\mathbb{C}}$ and $C \in A d K^{\mathbb{C}} \cdot \xi_{-1}$ with $[C, \bar{C}]=0$ so that

$$
h \# f_{C}=\left[\Phi_{\mu}\right] .
$$

Also in the general case (of not necessarily finite type harmonic maps) a natural class are those harmonic maps $\left[\Phi_{\mu}\right]$, which arise from holomorphic potentials

$$
\mu=\lambda^{-1} \eta,
$$

where $\eta$ is a $\mathfrak{p}^{\mathbb{C}}$-valued holomorphic 1-form on $M$. In particular, such harmonic maps $\left[\Phi_{\mu}\right]$ are given by finitely many holomorphic differentials. Interestingly enough, any harmonic map $f: M \rightarrow G / K$ may be obtained from a potential of the form (4.35) if one allows $\eta$ to be meromorphic.

To see this let $f: M \rightarrow G / K$ be harmonic with holomorphic potential $\mu \in \mathcal{P}$. Then (4.10) $f=\left[\Phi_{\mu}\right]$ where $g=\Phi_{\mu} b$ and $g: M \rightarrow \Lambda G_{\sigma}^{\mathbb{C}}$ integrates

$$
g^{-1} \partial g=\mu, \quad g(0)=e .
$$

Since $g: M \rightarrow \Lambda G_{\sigma}^{\mathbb{C}}$ is holomorphic and $\tau: \Lambda G_{\sigma}^{\mathbb{C}} \rightarrow D e t^{*}$ is holomorphic we know from Corollary 2.5 that the zero locus of $\tau \circ g$,

$$
S=\{p \in M ; g(p) \text { not contained in the big cell }\} \subset M,
$$

is discrete. But $g=\Phi_{\mu} b$ with $b$ taking values in $\Lambda_{B}^{+} G_{\sigma}^{\mathbb{C}}$, so that $g(p)$ is in the big cell if and only if $\Phi_{\mu}(p)$ is, in particular

$$
\begin{aligned}
& g_{-}=\left(\Phi_{\mu}\right)_{-}, \\
& S=\left(\tau \circ \Phi_{\mu}\right)^{-1}(0),
\end{aligned}
$$


and Lemma 2.6 implies that $g_{-}$extends meromorphically across $S$. Notice that if $F: M \rightarrow \Lambda G_{\sigma}$ is another extended lift for $f$ then (4.2) $\widetilde{F}=k \Phi_{\mu} H$ for some $k \in K$ and $H: M \rightarrow K$. Thus the set $S \subset M$ depends only on the harmonic map $f: M \rightarrow G / K$.

Theorem 4.10. Let $f: M \rightarrow G / K$ be harmonic with $f(0)=e K$ and $S \subset$ $M$ as defined above. Then there exists a $\mathfrak{p}^{\mathbb{C}}$-valued meromorphic 1-form $\eta$ on $M$ with poles contained in $S$ so that

$$
f=\left[\Phi_{\lambda^{-1} \eta}\right]
$$

on $M \backslash S$.

Proof. Let $\widetilde{F}: M \rightarrow \Lambda G_{\sigma}$ be an extended lift of $f$ with $\widetilde{F}(0)=e$. Then, on $M \backslash S$, we can decompose

$$
\widetilde{F}=F_{-} F_{+}
$$

where $F_{-}$takes values in $\Lambda_{*}^{-} G_{\sigma}^{\mathbb{C}}, F_{+}$takes values in $\Lambda^{+} G_{\sigma}^{\mathbb{C}}$ and $F_{-}$extends meromorphically across $S$ (4.36). But

$$
\begin{aligned}
F_{-}^{-1} d F_{-} & =\operatorname{Ad} F_{+}\left(\widetilde{F}^{-1} d \widetilde{F}\right)-d F_{+} F_{+}^{-1} \\
& =\operatorname{Ad} F_{+}\left(\lambda^{-1} \alpha_{1}^{\prime}+\alpha_{0}+\lambda \alpha_{1}^{\prime \prime}\right)-d F_{+} F_{+}^{-1} \\
& =\lambda^{-1} \operatorname{Ad}\left(F_{+}\right)_{0}\left(\alpha_{1}^{\prime}\right)=: \lambda^{-1} \eta,
\end{aligned}
$$

due to the direct sum decomposition $\Lambda \mathfrak{g}_{\sigma}^{\mathbb{C}}=\Lambda_{*}^{-} \mathfrak{g}_{\sigma}^{\mathbb{C}} \oplus \Lambda^{+} \mathfrak{g}_{\sigma}^{\mathbb{C}}$, where $\eta: M \rightarrow \mathfrak{p}^{\mathbb{C}}$ is meromorphic with poles contained in $S$. Moreover, $F_{-}(0)=e$ so that, together with (4.37), (4.8)

$$
F_{-}=g_{\lambda-1} \eta
$$

on $M \backslash S$. If we let

$$
F_{+}=b^{-1} H^{-1}
$$

with $H: M \backslash S \rightarrow K$ and $b: M \backslash S \rightarrow \Lambda_{B}^{+} G_{\sigma}^{\mathbb{C}}$, we obtain

$$
g_{\lambda^{-1} \eta}=F_{-}=\widetilde{F} F_{+}^{-1}=(\widetilde{F} H) b,
$$

so that by (4.10)

$$
\Phi_{\lambda^{-1} \eta}=\widetilde{F} H
$$

and thus

$$
f=\left[\Phi_{\lambda^{-1} \eta}\right] .
$$


We conclude this section with a discussion of how to apply the above theory to the construction of constant mean curvature surfaces with prescribed umbilic points. Let $\phi: M \rightarrow \mathbb{R}^{3}$ be a (simply connected) constant mean curvature surface and let $f: M \rightarrow S^{2}$ be its Gauss map. Then [25] $f$ is harmonic and the quadratic differential given by the $(2,0)$-part of the induced metric

$$
Q=(d f, d f)^{(2,0)}
$$

is holomorphic. The zeros of $Q$ are the umbilic points of the constant mean curvature surface $\phi: M \rightarrow \mathbb{R}^{3}$. Unless $M$ is the Riemann sphere, in which case $Q \equiv 0$ and $\phi(M) \subset \mathbb{R}^{3}$ is a round sphere, $f: M \rightarrow S^{2}$ is nonconformal. We view $S^{2}$ as the symmetric space $\boldsymbol{S} \boldsymbol{U}(2) / S^{1}$ with symmetric involution $\sigma(g)=s g s^{-1}, s=\left(\begin{array}{rr}1 & 0 \\ 0 & -1\end{array}\right)$, and Cartan decomposition $\mathfrak{g}=\mathfrak{k} \oplus \mathfrak{p}$ where

$$
\begin{aligned}
& \mathfrak{k}=\left\{\left(\begin{array}{cc}
i a & 0 \\
0 & -i a
\end{array}\right) ; \quad a \in \mathbb{R}\right\}, \\
& \mathfrak{p}=\left\{\left(\begin{array}{cc}
0 & b \\
-\bar{b} & 0
\end{array}\right) ; \quad b \in \mathbb{C}\right\} .
\end{aligned}
$$

If $F: M \rightarrow \boldsymbol{S U}(2)$ is a frame of $f$ then, (3.5),

$$
F^{-1} d F=\alpha_{0}+\alpha_{1}
$$

and by Definition 3.4, $\widetilde{F}: M \rightarrow \Lambda S U(2)_{\sigma}$ solving

$$
\widetilde{F}^{-1} d \widetilde{F}=\lambda^{-1} \alpha_{1}^{\prime}+\alpha_{0}+\lambda \alpha_{1}^{\prime \prime},
$$

is an extended lift. Notice that under the identification (3.2), (3.4)

$$
d f=\operatorname{Ad} F\left(\alpha_{1}\right)
$$

so that

$$
Q=\left(\alpha_{1}^{\prime}, \alpha_{1}^{\prime}\right),
$$

where $($,$) denotes the Killing form on \mathfrak{p}^{\mathbb{C}}$. From Lemma 4.5 we know that every harmonic map $f: M \rightarrow S^{2}$ is obtained from an extended lift $\widetilde{F}$ arising from a holomorphic potential $\mu=\sum_{k \geq-1} \lambda^{k} \mu_{k}$ via the decomposition (4.10)

$$
g_{\mu}=\widetilde{F} b
$$


where $g_{\mu}$ integrates $g^{-1} d g=\mu, g(0)=e$. Moreover, (4.40) and (4.13) imply that

$$
\alpha_{1}^{\prime}=\operatorname{Ad} b_{0}\left(\mu_{-1}\right),
$$

where $b_{0}=b_{\left.\right|_{\lambda=0}}: M \rightarrow B$. Thus, the holomorphic differential $Q$ is given by the product of the leading term $\mu_{-1}$ of the holomorphic potential $\mu$,

$$
Q=\left(\mu_{-1}, \mu_{-1}\right) .
$$

Note that by Theorem 4.10 one may assume that, away from a discrete set of points in $M, \mu=\lambda^{-1} \eta$ for a holomorphic $\mathfrak{p}^{\mathbb{C}}$-valued 1-form $\eta$. This suggests the following recipe to construct special constant mean curvature surfaces with prescribed umbilics: choose two holomorphic functions $a_{1}, a_{2}: M \rightarrow \mathbb{C}$ and consider the holomorphic potential

$$
\mu=\lambda^{-1}\left(\begin{array}{cc}
0 & a_{1} \\
a_{2} & 0
\end{array}\right) d z
$$

Then $\widetilde{F}: M \rightarrow \Lambda \boldsymbol{S U}(2)_{\sigma}$, where $g_{\mu}=\widetilde{F} b$, is an extended lift of the harmonic map $f=\pi \circ \widetilde{F}_{1}: M \rightarrow \boldsymbol{S U}(2) / S^{1}$. From Sym's formula [2] we know that $f$ is the Gauss map of the constant mean curvature surface

$$
\phi=\left(\frac{d}{d \theta}{ }_{\mid \theta=0} \widetilde{F}\right) \widetilde{F}^{-1}+f: M \rightarrow s u(2) \cong \mathbb{R}^{3}
$$

where $\lambda=e^{i \theta}$. Moreover, in view of (4.39), (4.41) and (4.42) one can show by computing the differential of $\phi$ that the umbilic points of $\phi$ are given by the zeros of $a_{2}$ and $\phi$ has branch points at the zeros of $a_{1}$. (The parallel constant mean curvature surface to $\phi$ is given by $\phi-2 f$ which has umbilics at the zeroes of $a_{1}$ and branch points at the zeros of $a_{2}$.) Hence, by selecting $a_{1}, a_{2}: M \rightarrow \mathbb{C}$ accordingly we can arrange for any prescribed umbilic configuration. A detailed study of constant mean curvature surfaces so obtained will be done elsewhere. We content ourselves here with some examples:

(i) $a_{1} \equiv 1, a_{2} \equiv 0, M=\mathbb{C}$, gives the round sphere minus a point;

(ii) $a_{1}=-a_{2} \equiv 1, M=\mathbb{C}$, gives the cylinder;

(iii) $a_{1}=1, a_{2} \equiv z^{m}, M=\mathbb{C}$, gives properly immersed constant mean curvature planes with one umbilic of order $m$ at the origin and rotational induced metric initially constructed by B. Smyth [27], [13], [2] (see Picture 1); 


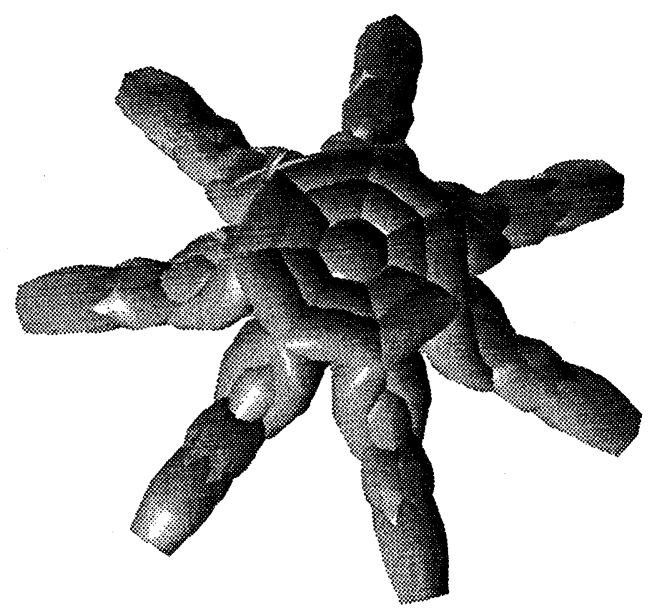

Figure 1: $a_{2}=z^{5}$.

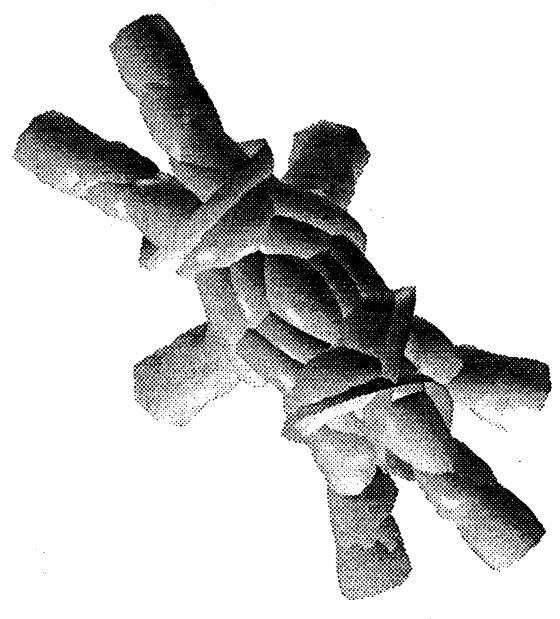

Figure 2: $a_{2}=\Pi_{k=1}^{5}\left(z-e^{2 \pi i k / 5}\right)$. 
(iv) $a_{1}=1, a_{2}=\Pi_{k=1}^{n}\left(z-p_{k}\right), p_{k} \in \mathbb{C}, M=\mathbb{C}$, gives the so called generalized Smyth surfaces with umbilics at $p_{k} \in \mathbb{C}$ (see Picture 2).

For the construction of compact constant mean curvature surfaces one expects that in general the holomorphic potential will have higher order terms in $\lambda$. Except for the leading term $\mu_{-1}$ we have no geometrical interpretation of those higher order terms. 


\section{Appendix: The $\bar{\partial}$-problem.}

Since we could not find a reference in the literature we give a brief outline how to solve the $\bar{\partial}$-problem in the setting of Lemma 4.5. Let $M$ be either $\mathbb{C}$ or the unit disc and $A+\lambda B: M \rightarrow \Lambda^{+} \mathcal{G}_{\sigma}^{\mathbb{C}}$ so that $A: M \rightarrow \mathfrak{k}^{\mathbb{C}}$ and $B: M \rightarrow \mathfrak{p}^{\mathbb{C}}$. Since $A$ and $B$ are the $(0,1)$-components of the derivative of a harmonic map they are real analytic on $M$. Consider the $\bar{\partial}$-problem

$$
\begin{aligned}
& g_{\bar{z}}=(A+\lambda B) g \\
& g(0)=1
\end{aligned}
$$

where $z$ is a global holomorphic coordinate on $M$.

Lemma 4.11. (4.43) has a (real analytic) solution $g: M \rightarrow \Lambda^{+} G_{\sigma}^{\mathbb{C}}$.

Proof. First we show that we have local solutions around every point $p_{0} \in M$. Since $A, B$ are real analytic there exist holomorphic maps

$$
\begin{aligned}
& \widetilde{A}: D_{\epsilon}\left(p_{0}\right) \times D_{\epsilon}\left(\bar{p}_{0}\right) \longrightarrow \mathfrak{k}^{\mathbb{C}} \\
& \widetilde{B}: D_{\epsilon}\left(p_{0}\right) \times D_{\epsilon}\left(\bar{p}_{0}\right) \longrightarrow \mathfrak{p}^{\mathbb{C}}
\end{aligned}
$$

where $D_{\epsilon}(p)$ is the $\epsilon$-disc around $p \in M$, so that

$$
\widetilde{A}(z, \bar{z})=A(z, \bar{z}), \widetilde{B}(z, \bar{z})=B(z, \bar{z}) .
$$

Consider the equation

$$
\begin{aligned}
& \widetilde{g}_{w}=(\widetilde{A}+\lambda \widetilde{B}) \widetilde{g} \\
& \widetilde{g}\left(z, \bar{p}_{0}\right)=1
\end{aligned}
$$

which has a unique (holomorphic) solution

$$
\widetilde{g}: D_{\epsilon}\left(p_{0}\right) \times D_{\epsilon}\left(\bar{p}_{0}\right) \rightarrow \Lambda^{+} G_{\sigma}^{\mathbb{C}} .
$$

Then $g: D_{\epsilon}\left(p_{0}\right) \rightarrow \Lambda^{+} G_{\sigma}^{\mathbb{C}}$ defined by

$$
g(z, \bar{z}):=\widetilde{g}(z, \bar{z})
$$

solves

$$
g_{\bar{z}}=(A+\lambda B) g
$$


Thus we have an open cover $\left(U_{\alpha}\right)$ of $M$ with (real analytic) solutions $g_{\alpha}: U_{\alpha} \rightarrow \Lambda^{+} G_{\sigma}^{\mathbb{C}}$ of

$$
g_{\bar{z}}=(A+\lambda B) g
$$

On the overlap $U_{\alpha} \cap U_{\beta}$ the maps

$$
h_{\alpha \beta}=g_{\alpha}^{-1} g_{\beta}: U_{\alpha} \cap U_{\beta} \rightarrow \Lambda^{+} G_{\sigma}^{\mathbb{C}}
$$

are holomorphic and satisfy the co-cycle condition

$$
h_{\alpha \beta} h_{\beta \gamma}=h_{\alpha \gamma} .
$$

But those are the data for a holomorphic $\Lambda^{+} G_{\sigma}^{\mathbb{C}}$-principal fiber bundle $P \rightarrow M$ whose (local) holomorphic sections are described by the $\bar{\partial}$-equation (4.43). Since $M$ is contractible $P$ admits a continuous global section. By a generalization of Grauert's Theorem [3] there exists a global holomorphic section $g: M \rightarrow \Lambda^{+} G_{\sigma}^{\mathbb{C}}$. To satisfy the initial condition take $g g(0)^{-1}$.

\section{References.}

[1] M. Adler and P. van Moerbeke, Completely integrable systems, Euclidean Lie algebras, and curves, Adv. Math. 38 (1980), 267-317.

[2] A.I. Bobenko, Constant mean curvature surfaces and integrable equations, Russian Math. Surveys 46:4 (1991), 1-45.

[3] L. Bungart, On analytic fiber bundles, Topology, 7 (1968), 55-68.

[4] F.E. Burstall, Harmonic tori in spheres and complex projective spaces, J. Reine Angew. Math. 469, 149-177 (1995).

[5] F.E. Burstall, D. Ferus, F. Pedit and U. Pinkall, Harmonic tori in symmetric spaces and commuting Hamiltonian systems on loop algebras, Ann. of Math., 138 (1993), 173-212.

[6] F.E. Burstall and F. Pedit, Dressing orbits of harmonic maps, Duke J. Math, Vol. 80, No.2, (1995), 353-382.

[7] F.E. Burstall and F. Pedit, Harmonic maps via Adler-Kostant-Symes theory in Harmonic Maps and Integrable Systems, Aspects of Mathematics, E23, Vieweg, A.P. Fordy and J. C. Wood, editors.

[8] F.E. Burstall and J.H. Rawnsley, Twistor theory for Riemannian symmetric spaces with applications to harmonic maps of Riemann surfaces, Lect. Notes in Math., vol. 1424, Springer, Berlin, Heidelberg, New York, 1990. 
[9] J. Dorfmeister, E. Neher and J. Szmigielski, Automorphisms of Banach manifolds associated with the KP-equation, Quart. J. Math. Oxford 40:2 (1989), 161-195.

[10] J. Dorfmeister and H. Wu, Constant mean curvature surfaces and loop groups, J. reine angew. Math. 440 (1993), 43-76.

[11] J. Eells and J.C. Wood, Harmonic maps from surfaces into projective spaces, Adv. in Math. 49 (1983), 217-263.

[12] D. Ferus, F. Pedit, U. Pinkall and I. Sterling, Minimal tori in $S^{4}$, J. reine angew. Math. 429 (1992), 1-47.

[13] D. Ferus, U. Pinkall and M. Timmreck, Constant mean curvature planes with inner rotational symmetry in Euclidean 3-space, TU-preprint, 1991, Berlin.

[14] M.A. Guest and Y. Ohnita, Loop group actions on harmonic maps and their applications in Harmonic Maps and Integrable Systems, Aspects of Mathematics, E23, Vieweg, A. P. Fordy and J. C. Wood, editors.

[15] S. Helgason, Differential geometry, Lie groups and symmetric spaces, Academic Press, Inc., 1978.

[16] N.J. Hitchin, The self-duality equations on a Riemann surface, Proc. Lond. Math. Soc. 55 (1987), 59-126.

[17] N.J. Hitchin, Harmonic maps from a 2-torus to the 3-sphere, J. Diff. Geom. 31 (1990), 627-710.

[18] N. Kapouleas, Compact constant mean curvature surfaces in Euclidean threespace, J. Diff. Geom. 33 (1991), 683-715.

[19] N. Kapouleas, Constant mean curvature surfaces by fusing Wente tori, Proc. Natl. Acad. Sci. USA 89 (1992), 5695-5698.

[20] H. B. Lawson, Complete minimal surfaces in $S^{3}$, Ann. of Math. 92 (1970), $335-374$.

[21] I. McIntosh, Global solutions of the elliptic 2D Toda lattice, preprint, 1992.

[22] U. Pinkall and I. Sterling, On the classification of constant mean curvature tori, Ann. of Math. 130 (1989), 407-451.

[23] A.N. Pressley and G. Segal, Loop Groups, Oxford Math. Monographs, Clarendon Press, Oxford, 1986.

[24] A.G. Reyman and M.A. Semenov-Tian-Shansky, Compatible Poisson structures for Lax equations: an r-matrix approach, Phys. Let. A 130 (1988), 456460. 
[25] E.A. Ruh and J. Vilms, The tension field of the Gauss map, Trans. Amer. Math.Soc. 149 (1970), 569-573.

[26] G. Segal and G. Wilson, Loop groups and equations of KdV type, Pub. Math. de I.H.E.S. 61 (1985), 5-65.

[27] B. Smyth, The generalization of Delaunay's Theorem to constant mean curvature surfaces with continuous internal symmetry, preprint 1987.

[28] W. Symes, Systems of Toda type, inverse spectral problems and representation theory, Invent. Math. 159 (1980), 13-51.

[29] K. Uhlenbeck, Harmonic maps into Lie groups (classical solutions of the chiral model), J. Diff. Geom. 30 (1989), 1-50.

[30] H.C. Wente, Counterexample to a conjecture of H. Hopf, Pac. J. Math. 121 (1986), 193-243.

[31] $\mathrm{H}$. Wu, Banach manifolds of minimal surfaces in the 4-sphere, Differential Geometry (R.E. Greene and S.T. Yau, eds.), Proc. Symp. Pure Math., vol. 54, Amer. Math. Soc., Providence RI, 1993, pp. 530-539.

[32] V.E. Zakharov and A.B. Shabat, Integration of non-linear equations of mathematical physics by the method of inverse scattering, II, Funkts. Anal i Prilozhen 13 (1978), 13-22.

Received July 26, 1995.

UNIVERSITY OF KANSAS

LAWRENCE, KANSAS 66045

E-mail address: DORFMEISTER@KUHUB.CC.UKANS.EDU

UNIVERSiTY OF MASSACHUSETTS

AMHERST, MA 01003

E-mail address: FRANZ@GANG.UMASS.EDU

AND

NORTHERn IlLINOIS UnIVERSITY

DeKaLB, IL 60115

E-mail address: WU@MATH.NIU.EDU 